\title{
Design and synthesis of novel
} as potential fungicides

\author{
Xiaobin Wang ${ }^{1}$, Zhengjiao Ren ${ }^{1}$, Mengqi Wang ${ }^{1}$, Min Chen ${ }^{1}$, Aiming Lu' ${ }^{1}$, Weijie Si ${ }^{1,2}$ and Chunlong Yang ${ }^{1,2^{*}}$
}

\begin{abstract}
Background: Tetramic acid, thiophene and hydrazone derivatives were found to exhibit favorable antifungal activity. Aiming to discover novel template molecules with potent antifungal activity, a series of novel 3-(thiophen-2-yl)-1,5-dihydro-2H-pyrrol-2-one derivatives containing a hydrazone group were designed, synthesized, and evaluated for their antifungal activity.

Results: The structures of 3-(thiophen-2-yl)-1,5-dihydro-2H-pyrrol-2-one derivatives bearing a hydrazone group were confirmed by FT-IR, ${ }^{1} \mathrm{H}$ NMR, ${ }^{13} \mathrm{C}$ NMR, ${ }^{1} \mathrm{H}-{ }^{1} \mathrm{H}$ NOESY, EI-MS and elemental analysis. Antifungal assays indicated that some title compounds exhibited antifungal activity against Fusarium graminearum (Fg), Rhizoctorzia solani (Rs), Botrytis cinerea $(B C)$ and Colletotrichum capsici $(C c)$ in vitro. Strikingly, the $E_{50}$ value of $\mathbf{5 e}$ against $R s$ was $1.26 \mu \mathrm{g} / \mathrm{mL}$, which is better than that of drazoxolon $(1.77 \mu \mathrm{g} / \mathrm{mL})$. Meanwhile, title compounds $\mathbf{5 b}, \mathbf{5 d}, \mathbf{5 e}-\mathbf{5 g}, \mathbf{5 n} \mathbf{- 5 q}$ and $\mathbf{5 t}$ exhibited remarkable anti-Cc activity, with corresponding $\mathrm{EC}_{50}$ values of 7.65, 9.97, 6.04, 6.66, 7.84, 7.59, 9.47, 5.52, 6.41 and $7.53 \mu \mathrm{g} / \mathrm{mL}$, respectively, which are better than that of drazoxolon $(19.46 \mu \mathrm{g} / \mathrm{mL})$.

Conclusions: A series of 3-(thiophen-2-yl)-1,5-dihydro-2H-pyrrol-2-one derivatives bearing a hydrazone group were designed, synthesized and evaluated for their antifungal activity against $F g, R s, B C$ and $C c$. Bioassays indicated that some target compounds exhibited obvious antifungal activity against the above tested fungi. These results provide a significant basis for the further structural optimization of tetramic acid derivatives as potential fungicides.
\end{abstract}

Keywords: Tetramic acid, Hydrazone, Thiophene, Synthesis, Antifungal activity

\section{Background}

An emergence of resistant fungi is a huge impetus to the development of agricultural fungicides with novel molecular structures and unique mechanisms [1]. In this regard, the structural optimization of natural heterocycles plays a important role in the searching for bioactive lead compounds [2, 3]. As attractive nitrogenous heterocycles, tetramic acid derivatives are widely researched for some reasons. First, tetramic acid derivatives exist in secondary metabolites from

\footnotetext{
*Correspondence: ycl@njau.edu.cn

1 Jiangsu Key Laboratory of Pesticide Science, College of Sciences,

Nanjing Agricultural University, Nanjing 210095, China

Full list of author information is available at the end of the article
}

various terrestrial and marine organisms and have favorable compatibility with the environment [4]. Second, tetramic acid derivatives contain a unique pyrroline-2-one or pyrrolidine-2,4-dione substructure that is easy to synthesize to some extent [5]. Third, tetramic acid derivatives are reported to exhibit various agricultural bioactivities including fungicidal [6], herbicidal [7], insecticidal [8], antibacterial and antiviral [9] properties. Encouraged by the above findings, series of tetramic acid derivatives bearing amino [10], strobilurin [6], phenylhydrazine [11], oxime ether [12] and pyrrole [13] groups were synthesized and reported for their antifungal activity against plant fungi in our previous work. However, the potential application of 
tetramic acid derivatives as agricultural fungicides was greatly limited by their unsatisfactory curative rates $[6$, 10-13].

Thiophene is an important sulphureous compound that was widely studied for the development of novel fungicides due to their wide and satisfactory antifungal activity [14-17]. As important thiophene derivatives, thicyofen, ethaboxam, silthiopham and penthiopyrad were commercialized as agricultural fungicides in the past decades. Meanwhile, hydrazone is a widely researchful substructure that exists in commercialized agrochemicals including ferimzone, hydramethylnon, diflufenzopyr, pymetrozine, metaflumizone and benquinox [18, 19]. Recently, scholars found introducing a hydrazone group into salicylaldehyde [20], nalidixic acid [21], tetrahydro- $\beta$-carboline [22], 1,2,3-triazole [23], benzimidazole [24], diphenyl ether [25], pyrazole amide [26] quinoxaline [27] and carbonic acid ester [28] could effectively improve and broaden their antifungal activity. Obviously, further structural modifications of thiophene and hydrazone derivatives are significant for the development of novel fungicides.

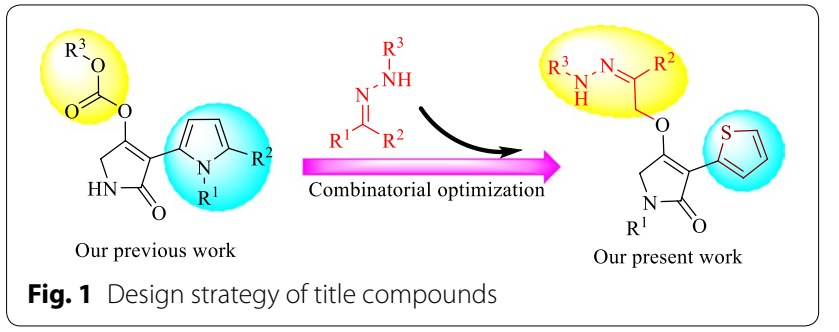

Aiming to extend our previous works on searching for pyrroline-2-one derivatives as agricultural fungicides $[6,10-13,29]$, we theorized that introducing a hydrazone group into pyrroline-2-one structure might generate novel lead molecules with better antifungal activity (Fig. 1). Thus, in this study, a thiophene group was firstly neatly combined with pyrroline-2-one scaffold in one molecule by a Dieckmann cyclization. Subsequently, a hydrazone group was introduced into the 4-position of the obtained 3-(thiophen-2-yl)-1,5-dihydro- $2 H$-pyrrol2-one substructure to generate a series of novel tetramic acid derivatives (Scheme 1). In addition, the fungi Fusarium graminearum (Fg), Rhizoctorzia solani (Rs), Botrytis cinerea $(B c)$ and Colletotrichum capsici $(C c)$, which seriously restricted agricultural outputs of wheat, rice, strawberries and pepper, were selected as tested fungi to evaluate the antifungal activity of 3-(thiophen-2-yl)1,5-dihydro- $2 H$-pyrrol-2-one derivatives bearing a hydrazone group. To the best of our knowledge, it is the first report on the synthesis and antifungal activity of 3-(thiophen-2-yl)-1,5-dihydro- $2 \mathrm{H}$-pyrrol-2-one derivatives bearing a hydrazone group.

\section{Results and discussion Chemistry}

The synthetic route to 3-(thiophen-2-yl)-1,5-dihydro- $2 \mathrm{H}$-pyrrol-2-one derivatives containing a hydrazone group is shown in Scheme 1. Using a substituted glycine ethyl ester hydrochloride as a starting material, the key intermediate 2 (substituted 4-hydroxy-3-(thiophen-2-yl)-1,5-dihydro-2H-pyrrol-2one) was synthesized by two steps including amidation

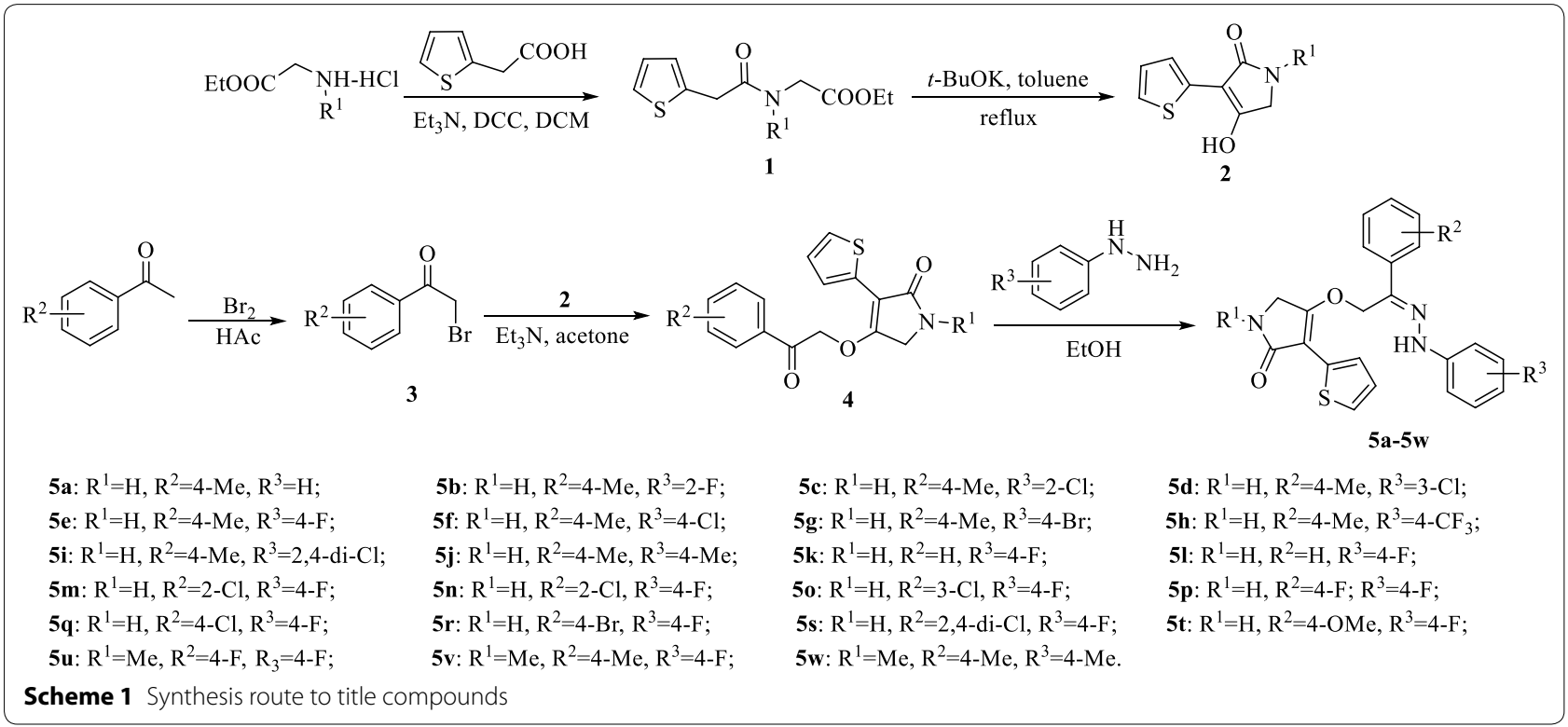


and cyclization reactions. The intermediate 2 was reacted with substituted 2-bromo-1-phenylethan1 -one 3 in acetone containing triethylamine to obtain the substituted 4-(2-oxo-2-phenylethoxy)-3-(thiophen2-yl)-1,5-dihydro-2H-pyrrol-2-one 4. Subsequently, the obtained intermediate 4 was reacted with substituted phenylhydrazine in acetonitrile to yield the title compound $\mathbf{5}$ with a good yield. The structures of title compounds were confirmed by FT-IR, ${ }^{1} \mathrm{H}$ NMR, ${ }^{13} \mathrm{C}$ NMR, EI-MS, and elemental analysis. In the IR spectra of title compounds, two obvious peaks at 32943447 and $3171-3263 \mathrm{~cm}^{-1}$ are attributed to the $\mathrm{N}-\mathrm{H}$ stretching vibrations at pyrroline-2-one and phenylhydrazone fragments. The absorption peak of the carbonyl group at 2-position of pyrroline-2-one appears at $1682-1667 \mathrm{~cm}^{-1}$. In ${ }^{1} \mathrm{H}$ NMR spectra, two singlets at $\delta$ 9.12-10.35 and 7.83-8.00 ppm are assigned to the $\mathrm{NH}$ protons at phenylhydrazone and pyrroline-2-one fragments. Two singlets at $\delta 4.26-4.49$ and $5.36-5.58 \mathrm{ppm}$ mean that the structure of title compounds has two $-\mathrm{CH}_{2}-$ fragments. A typical carbon resonance at $\delta$ 169.51-172.01 ppm in the ${ }^{13} \mathrm{C}$ NMR spectra confirms the presence of a carbonyl group at 2-position of pyrroline-2-one. Meanwhile, singlets at 43.51-43.77 and 61.73-66.02 ppm confirm the existence of two $-\mathrm{CH}_{2}-$ fragments in the molecular structure of title compounds. In the EI-MS spectra of title compounds, the value of $[\mathrm{M}]^{+}$ion absorption signal is consistent with the calculated value of molecular weight.

\section{Configuration confirmation of title compounds}

As shown in the ${ }^{1} \mathrm{H}$ NMR and ${ }^{13} \mathrm{C}$ NMR spectra of title compounds, these 3-(thiophen-2-yl)-1,5-dihydro-2 $\mathrm{H}$-pyrrol-2-one derivatives containing a hydrazone group does present itself via one single molecular structure. Aiming to further understand the structural characteristics of title compounds, the configuration of compound $5 \mathbf{f}$ was studied as an example by a ${ }^{1} \mathrm{H}-{ }^{1} \mathrm{H}$ NOESY analysis [30]. As shown in Fig. 2, the chemical shifts of $\mathrm{H}_{\mathrm{f}}, \mathrm{H}_{\mathrm{j}}$ and $\mathrm{H}_{\mathrm{k}}$ protons were 5.39, 10.10 and $7.26 \mathrm{ppm}$ in the NOESY spectrum of compound $\mathbf{5 f}$ (DMSO- $d_{6}$ ), respectively. The obvious NOE phenomena between $H_{j}$ and $H_{f}$, and between $H_{j}$ and $H_{k}$ indicated that these protons close with each other, which typically revealed the double bond $\mathrm{C}=\mathrm{NNH}$ of title compound $\mathbf{5 f}$ possesses the cis-configuration.

\section{Antifungal activity screening of title compounds}

Using a mycelial growth rate method [6, 10-13, 21-28], the antifungal effects of title compounds $\mathbf{5 a}-\mathbf{5 w}$ against $R s, B c, C c$ and $F g$ were evaluated at $10 \mu \mathrm{g} / \mathrm{mL}$ and are shown in Table 1. A agricultural fungicide drazoxolon was used as a positive control of antifungal effects under same conditions. As shown in Table 1, the compounds 5n, 5p and $\mathbf{5 u}$ exhibited fine activity against $R s$, with inhibitory rates of $91.5,100.0$ and $84.7 \%$, respectively, which are better than that of drazoxolon (84.5\%). The compounds $\mathbf{5 g}, \mathbf{5 p}$ and $\mathbf{5 t}$ obviously inhibited the mycelium growth of $B c$, with inhibitory rates of 66.4, 61.1 and $51.3 \%$, respectively. The inhibition rates of compounds

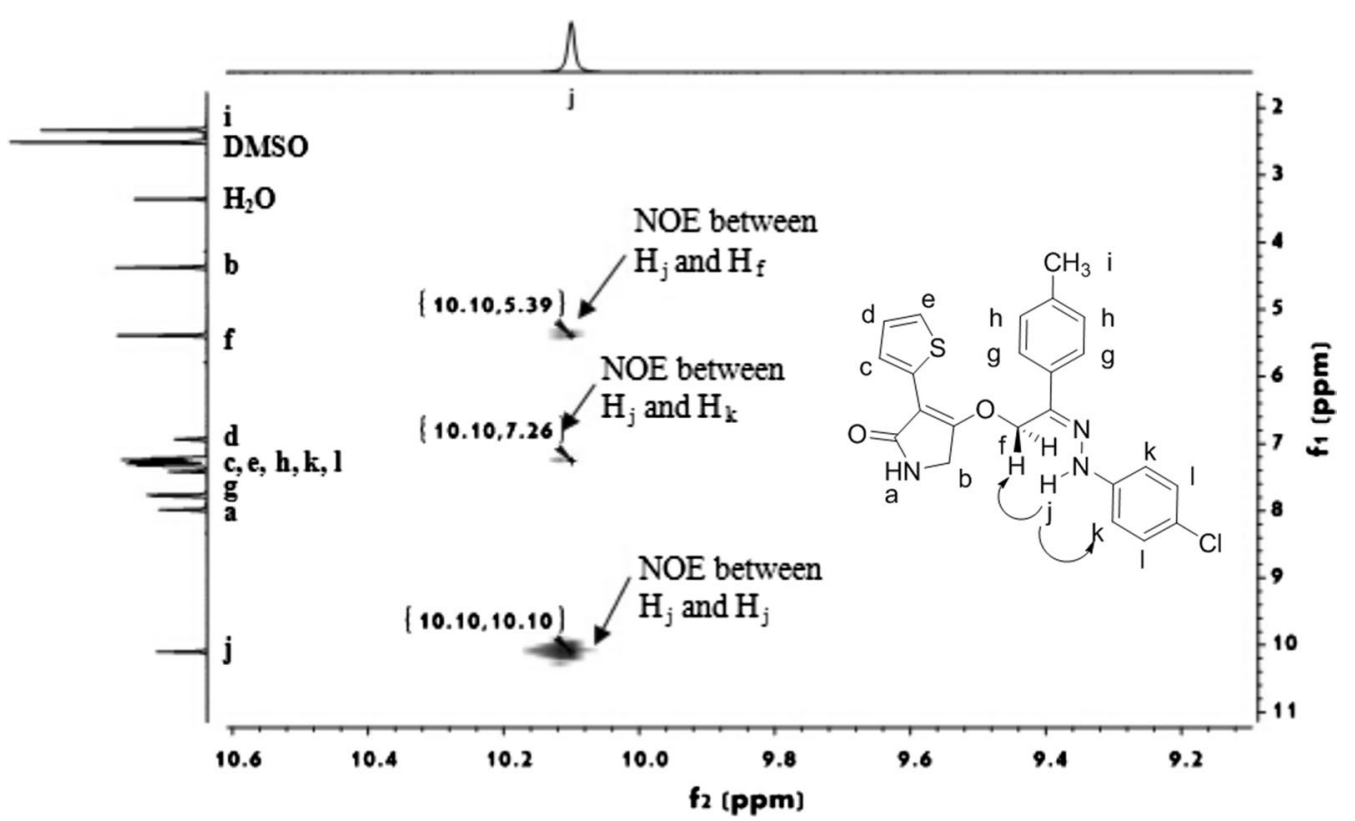

Fig. 2 NOESY spectrum of the title compound $\mathbf{5 f}$ 
Table 1 Antifungal effects of title compounds $5 \mathrm{a}-5 \mathrm{w}$ at $10 \mu \mathrm{g} / \mathrm{mL}$

\begin{tabular}{|c|c|c|c|c|c|c|c|}
\hline Compd. & $\mathrm{R}^{1}$ & $R^{2}$ & $R^{3}$ & Rs & $B C$ & $C c$ & $\mathrm{Fg}$ \\
\hline $5 a$ & $\mathrm{H}$ & $4-\mathrm{CH}_{3}$ & $\mathrm{H}$ & $10.5 \pm 0.5$ & $0.0 \pm 8.2$ & $16.4 \pm 2.6$ & $7.5 \pm 0.5$ \\
\hline $5 b$ & $\mathrm{H}$ & $4-\mathrm{CH}_{3}$ & $2-F$ & $67.7 \pm 0.5$ & $32.3 \pm 0.9$ & $57.3 \pm 2.7$ & $28.4 \pm 1.1$ \\
\hline $5 c$ & $\mathrm{H}$ & $4-\mathrm{CH}_{3}$ & $2-\mathrm{Cl}$ & $12.6 \pm 1.7$ & $25.3 \pm 1.8$ & $21.1 \pm 1.3$ & $12.2 \pm 1.7$ \\
\hline $5 d$ & $\mathrm{H}$ & $4-\mathrm{CH}_{3}$ & $3-\mathrm{Cl}$ & $70.6 \pm 2.1$ & $49.0 \pm 1.6$ & $61.3 \pm 1.8$ & $40.6 \pm 2.3$ \\
\hline $5 e$ & $\mathrm{H}$ & $4-\mathrm{CH}_{3}$ & $4-F$ & $83.9 \pm 1.2$ & $49.5 \pm 2.2$ & $90.7 \pm 1.7$ & $98.6 \pm 0.5$ \\
\hline $5 f$ & $\mathrm{H}$ & $4-\mathrm{CH}_{3}$ & $4-\mathrm{Cl}$ & $76.7 \pm 1.5$ & $34.4 \pm 0.5$ & $74.7 \pm 1.2$ & $69.0 \pm 0.6$ \\
\hline $5 g$ & $\mathrm{H}$ & $4-\mathrm{CH}_{3}$ & $4-B r$ & $66.7 \pm 0.6$ & $66.4 \pm 2.1$ & $74.7 \pm 1.9$ & $67.4 \pm 1.7$ \\
\hline $5 \mathrm{~h}$ & $\mathrm{H}$ & $4-\mathrm{CH}_{3}$ & $4-\mathrm{CF}_{3}$ & $44.1 \pm 3.0$ & $38.4 \pm 2.4$ & $43.2 \pm 2.1$ & $42.3 \pm 1.2$ \\
\hline $5 i$ & $\mathrm{H}$ & $4-\mathrm{CH}_{3}$ & 2,4-di-Cl & $47.5 \pm 2.4$ & $40.2 \pm 3.1$ & $18.9 \pm 2.7$ & $10.8 \pm 3.5$ \\
\hline $5 j$ & $\mathrm{H}$ & $4-\mathrm{CH}_{3}$ & $4-\mathrm{CH}_{3}$ & $40.7 \pm 1.8$ & $27.8 \pm 0.7$ & $38.9 \pm 1.8$ & $40.5 \pm 1.9$ \\
\hline $5 k$ & $\mathrm{H}$ & $4-\mathrm{CH}_{3}$ & $4-\mathrm{OCH}_{3}$ & $20.0 \pm 3.5$ & $7.3 \pm 1.4$ & $25.1 \pm 1.0$ & $9.4 \pm 2.8$ \\
\hline 51 & $\mathrm{H}$ & $\mathrm{H}$ & $4-\mathrm{F}$ & $52.8 \pm 1.1$ & $30.5 \pm 3.2$ & $40.7 \pm 2.1$ & $38.1 \pm 2.1$ \\
\hline $5 \mathrm{~m}$ & $\mathrm{H}$ & $2-\mathrm{Cl}$ & $4-F$ & $66.3 \pm 1.4$ & $37.5 \pm 2.2$ & $50.2 \pm 2.8$ & $47.3 \pm 1.3$ \\
\hline $5 n$ & $\mathrm{H}$ & $2-B r$ & $4-F$ & $91.5 \pm 2.0$ & $47.2 \pm 1.0$ & $93.0 \pm 1.3$ & $53.1 \pm 1.1$ \\
\hline 50 & $\mathrm{H}$ & $3-\mathrm{Cl}$ & $4-\mathrm{F}$ & $70.2 \pm 1.8$ & $36.1 \pm 0.9$ & $73.8 \pm 1.2$ & $74.6 \pm 3.2$ \\
\hline $5 p$ & $\mathrm{H}$ & $4-\mathrm{F}$ & $4-\mathrm{F}$ & $100.0 \pm 0.3$ & $61.1 \pm 3.5$ & $100.0 \pm 0.2$ & $100.0 \pm 0.3$ \\
\hline $5 q$ & $\mathrm{H}$ & $4-\mathrm{Cl}$ & $4-\mathrm{F}$ & $76.4 \pm 0.5$ & $37.5 \pm 1.4$ & $83.8 \pm 1.6$ & $68.6 \pm 2.3$ \\
\hline $5 r$ & $\mathrm{H}$ & $4-B r$ & $4-F$ & $55.0 \pm 1.8$ & $34.7 \pm 2.2$ & $48.5 \pm 1.6$ & $67.6 \pm 3.0$ \\
\hline $5 s$ & $\mathrm{H}$ & 2,4-di-Cl & $4-F$ & $70.6 \pm 3.3$ & $45.8 \pm 1.6$ & $43.8 \pm 3.0$ & $47.8 \pm 1.6$ \\
\hline $5 t$ & $\mathrm{H}$ & $4-\mathrm{OCH}_{3}$ & $4-F$ & $67.5 \pm 1.2$ & $51.3 \pm 2.9$ & $86.0 \pm 3.6$ & $92.7 \pm 2.5$ \\
\hline $5 u$ & $4-\mathrm{CH}_{3}$ & $4-\mathrm{F}$ & $4-\mathrm{F}$ & $84.7 \pm 1.1$ & $48.5 \pm 2.1$ & $53.8 \pm 3.3$ & $47.9 \pm 1.4$ \\
\hline $5 v$ & $4-\mathrm{CH}_{3}$ & $4-\mathrm{CH}_{3}$ & $4-\mathrm{F}$ & $64.7 \pm 2.1$ & $36.3 \pm 1.8$ & $36.1 \pm 1.1$ & $39.6 \pm 3.1$ \\
\hline $5 w$ & $4-\mathrm{CH}_{3}$ & $4-\mathrm{CH}_{3}$ & $4-\mathrm{CH}_{3}$ & $37.1 \pm 0.1$ & $25.8 \pm 0.4$ & $31.2 \pm 1.1$ & $35.2 \pm 2.1$ \\
\hline Drazoxolon $^{a}$ & / & / & / & $84.5 \pm 1.8$ & $91.2 \pm 2.2$ & $46.8 \pm 1.9$ & $67.2 \pm 0.9$ \\
\hline
\end{tabular}

Average of three replicates

a A commercial agricultural fungicide drazoxolon was used for comparison of antifungal activity

$\mathbf{5 b}, \mathbf{5 d}-\mathbf{5 g}, \mathbf{5 m}-\mathbf{5 r}, \mathbf{5 t}$ and $\mathbf{5 u}$ against $C c$ ranged from 48.5 to $100.0 \%$, which are better than that of drazoxolon (46.8\%). Table 1 also shown that the anti- $F g$ effects of target compounds $\mathbf{5 e}-\mathbf{5 g}, \mathbf{5 o}-\mathbf{5 r}$ and $\mathbf{5 t}$ at $10 \mu \mathrm{g} / \mathrm{mL}$ were 98.6, 69.0, 67.4, 74.6, 100.0, 68.6, 67.6 and 92.7\%, respectively, which are apparently better than that of drazoxolon (67.2\%).

Encouraged by the above preliminary bioassays, the $\mathrm{EC}_{50}$ values of some compounds that exhibited fine antifungal activity against $R s, C c$ and $F g$ at $10 \mu \mathrm{g} /$ $\mathrm{mL}$ were determined and are summarized in Table 2 . Table 2 shown that the $\mathrm{EC}_{50}$ values of the selected compounds ranged from 1.26 to $9.89 \mu \mathrm{g} / \mathrm{mL}$ against $R s$, from 5.52 to $9.97 \mu \mathrm{g} / \mathrm{mL}$ against $C c$ and from 6.02 to $8.85 \mu \mathrm{g} / \mathrm{mL}$ against $\mathrm{Fg}$. Strikingly, the $\mathrm{EC}_{50}$ value of the title compound 5e against $R s$ was $1.26 \mu \mathrm{g} / \mathrm{mL}$, which is better than that of drazoxolon $(1.77 \mu \mathrm{g} / \mathrm{mL})$. Meanwhile, the title compounds $\mathbf{5 b}, \mathbf{5 d}, \mathbf{5 e}-\mathbf{5 g}, \mathbf{5 n}-\mathbf{5 q}$ and 5t had remarkable $\mathrm{EC}_{50}$ values of 7.65, 9.97, 6.04, 6.66, 7.84, 7.59, 9.47, 5.52, 6.41 and $7.53 \mu \mathrm{g} / \mathrm{mL}$ against $C c$, respectively, which are better than that of drazoxolon $(19.46 \mu \mathrm{g} / \mathrm{mL})$. The above results also indicates that
3-(thiophen-2-yl)-1,5-dihydro-2H-pyrrol-2-one derivatives containing a hydrazone group can serve as potential structural templates in the search for novel and highly efficient fungicides.

\section{Structure-activity relationships}

As indicated in Tables 1 and 2, the antifungal effects of title compounds were greatly affected by structural variations. Some structure-activity relationships (SAR) analyses were discussed as below. First, Tables 1 and 2 show that most of title compounds exhibited better antifungal activity against $R s$ than that against $B c, C c$ and $F g$. For example, Table 1 presents that the anti-Rs effects of title compounds $\mathbf{5 b}, \mathbf{5 d}, \mathbf{5 f}, \mathbf{5 h}, \mathbf{5 i}, \mathbf{5 j}, \mathbf{5 l}, \mathbf{5 m}, \mathbf{5 p}, \mathbf{5 s}$, $\mathbf{5 u}, \mathbf{5 v}$ and $\mathbf{5 w}$ are better than the corresponding effects against $B c, C c$ and $F g$ at $10 \mu \mathrm{g} / \mathrm{mL}$. Table 2 also exhibits that title compounds $5 \mathbf{b}, 5 \mathbf{d}, 5 \mathbf{5 e}, \mathbf{5 f}, 5 \mathrm{~g}, 5 \mathbf{n}, 5 \mathbf{5}, 5 \mathbf{p}$ and 5q have better $\mathrm{EC}_{50}$ values against $R s$ than that against $\mathrm{Cc}$ and $\mathrm{Fg}$. Second, introducing methyl into the $\mathrm{R}^{1}$ position is disadvantageous for the antifungal activity of title compounds against the tested four fungi. For instance, Table 1 shows that the inhibition rates of compounds $\mathbf{5 e}$, 
Table $2 \mathrm{EC}_{50}$ values of some title compounds against $R s$, $\mathrm{CC}$ and $\mathrm{Fg}$

\begin{tabular}{|c|c|c|c|c|}
\hline Compd. & Tested fungus & $\begin{array}{l}\text { Regression } \\
\text { equation }\end{array}$ & $\mathbf{R}$ & $\mathrm{EC}_{50}(\mu \mathrm{g} / \mathrm{mL})$ \\
\hline \multirow[t]{2}{*}{$5 b$} & Rs & $y=0.76 x+4.73$ & 0.99 & $2.28 \pm 3.00$ \\
\hline & Cc & $y=0.81 x+4.28$ & 0.95 & $7.65 \pm 5.31$ \\
\hline \multirow[t]{2}{*}{$5 d$} & Rs & $y=1.42 x+3.57$ & 0.98 & $5.23 \pm 3.74$ \\
\hline & Cc & $y=1.60 x+2.95$ & 0.98 & $9.97 \pm 8.90$ \\
\hline \multirow[t]{3}{*}{$5 e$} & Rs & $y=0.87 x+4.91$ & 0.99 & $1.26 \pm 1.12$ \\
\hline & Cc & $y=1.42 x+3.89$ & 0.99 & $6.04 \pm 5.35$ \\
\hline & $\mathrm{Fg}$ & $y=2.32 x+3.17$ & 0.97 & $6.13 \pm 4.49$ \\
\hline \multirow[t]{3}{*}{$5 f$} & Rs & $y=0.50 x+4.74$ & 0.99 & $3.32 \pm 2.74$ \\
\hline & Cc & $y=1.25 x+3.97$ & 0.99 & $6.66 \pm 5.33$ \\
\hline & $\mathrm{Fg}$ & $y=1.74 x+3.54$ & 0.99 & $6.90 \pm 4.96$ \\
\hline \multirow[t]{3}{*}{$5 g$} & Rs & $y=0.38 x+4.77$ & 0.96 & $4.13 \pm 2.83$ \\
\hline & CC & $y=1.32 x+3.82$ & 0.99 & $7.84 \pm 7.03$ \\
\hline & $\mathrm{Fg}$ & $y=1.25 x+3.87$ & 0.96 & $8.03 \pm 5.01$ \\
\hline \multirow[t]{2}{*}{$5 n$} & Rs & $y=1.26 x+4.31$ & 0.99 & $3.56 \pm 3.16$ \\
\hline & Cc & $y=1.35 x+3.81$ & 0.97 & $7.59 \pm 5.12$ \\
\hline \multirow[t]{3}{*}{50} & Rs & $y=1.42 x+3.79$ & 0.98 & $7.15 \pm 5.62$ \\
\hline & Cc & $y=1.47 x+3.56$ & 0.99 & $9.47 \pm 8.02$ \\
\hline & $\mathrm{Fg}$ & $y=1.97 x+3.10$ & 0.99 & $7.22 \pm 6.01$ \\
\hline \multirow[t]{3}{*}{$5 p$} & Rs & $y=2.41 x+3.49$ & 0.99 & $2.22 \pm 1.68$ \\
\hline & Cc & $y=4.22 x+1.87$ & 0.99 & $5.52 \pm 5.49$ \\
\hline & $\mathrm{Fg}$ & $y=3.56 x+2.04$ & 0.98 & $6.77 \pm 5.14$ \\
\hline \multirow[t]{3}{*}{$5 q$} & Rs & $y=1.76 x+3.73$ & 0.99 & $5.29 \pm 4.54$ \\
\hline & Cc & $y=1.68 x+3.29$ & 0.99 & $6.41 \pm 4.96$ \\
\hline & $\mathrm{Fg}$ & $y=3.79 x+1.30$ & 0.99 & $7.63 \pm 5.81$ \\
\hline \multirow[t]{2}{*}{$5 r$} & Rs & $y=1.13 x+3.70$ & 0.98 & $9.89 \pm 7.18$ \\
\hline & $\mathrm{Fg}$ & $y=1.33 x+3.47$ & 0.99 & $8.85 \pm 8.26$ \\
\hline \multirow[t]{3}{*}{$5 t$} & Rs & $y=1.27 x+3.81$ & 0.99 & $8.62 \pm 7.06$ \\
\hline & Cc & $y=1.39 x+3.24$ & 0.98 & $7.53 \pm 6.89$ \\
\hline & $\mathrm{Fg}$ & $y=1.37 x+3.85$ & 0.97 & $6.02 \pm 5.26$ \\
\hline \multirow[t]{3}{*}{ Drazoxolon $^{\mathrm{a}}$} & Rs & $y=2.54 x+4.37$ & 0.99 & $1.77 \pm 1.62$ \\
\hline & Cc & $y=0.82 x+3.94$ & 0.99 & $19.46 \pm 3.93$ \\
\hline & $\mathrm{Fg}$ & $y=2.04 x+3.88$ & 0.99 & $3.53 \pm 2.72$ \\
\hline
\end{tabular}

Average of three replicates

a A commercial agricultural fungicide drazoxolon was used for comparison of antifungal activity

$5 \mathbf{j}$ and $5 \mathbf{p}\left(\mathrm{R}^{1}=\mathrm{H}\right)$ are obviously better than that of compounds $5 \mathbf{v}, \mathbf{5} \mathbf{w}$ and $\mathbf{5 u}\left(\mathrm{R}^{1}=\mathrm{Me}\right)$ against the tested four fungi at $10 \mu \mathrm{g} / \mathrm{mL}$. Third, when the $R^{2}$ was substituted by $4-\mathrm{Me}, 4-\mathrm{F}, 2-\mathrm{Br}$ and $4-\mathrm{OMe}$ groups, the corresponding title compounds $\mathbf{5 e}, \mathbf{5 n}, \mathbf{5 p}$ and $\mathbf{5 t}$ exhibited overall better antifungal activity than that of compounds $\mathbf{5 1}, \mathbf{5 m}$, 50 and $\mathbf{5 q}-5 \mathrm{~s}$ against $R s, B c$ and $F g$ at $10 \mu \mathrm{g} / \mathrm{mL}$. Finally, a presence of $4-\mathrm{F}, 4-\mathrm{Cl}$ and $4-\mathrm{Br}$ groups at the $\mathrm{R}^{3}$ position can effectively enhance the antifungal activity of title compounds against $R s, B c$ and $F g$. For example, the inhibition effects of compounds $5 \mathbf{e}, \mathbf{5 f}$ and $\mathbf{5 g}$ were overall better than that of compounds $\mathbf{5 a}-\mathbf{5 d}$ and $\mathbf{5 h}-\mathbf{5} \mathbf{k}$ against $R s, B c$ and $F g$ at $10 \mu \mathrm{g} / \mathrm{mL}$.

\section{Methods and materials \\ General}

Reagents and solvents used without further purification are analytically or chemically pure. Melting points (m.p.) were determined on an uncorrected WRS-1B digital melting point apparatus (Shanghai Precision and Scientific Instrument Corporation, China). The FT-IR spectra were recorded on a Thermo Nicolet 380 FT-IR spectrometer (Thermo Nicolet Corporation, America). ${ }^{1} \mathrm{H}$ NMR, ${ }^{13} \mathrm{C}$ NMR, and ${ }^{1} \mathrm{H}_{-}{ }^{1} \mathrm{H}$ NOESY spectra were collected on a Bruker AV $400 \mathrm{MHz}$ spectrometer (Bruker Corporation, Germany) at room temperature with DMSO- $d_{6}$ as a solvent. Mass spectra were recorded on a TRACE 2000 spectrometer (Finnigan Corporation, America). Elemental analyses were determined on an Elementar Vario EL cube analyzer (Elementar Corporation, German). Reactions were monitored by thin layer chromatography (TLC) on silica gel $\mathrm{GF}_{245}$ (400 mesh). The tested strains $\mathrm{Fg}, \mathrm{Rs}, \mathrm{Bc}$ and $\mathrm{Cc}$ were provided by the Laboratory of Plant Disease Control at Nanjing Agricultural University.

\section{General procedures for intermediates $\mathbf{2}$ and $\mathbf{3}$}

Using glycine ethyl ester hydrochloride or alanine ethyl eater hydrochloride as a starting material, the intermediate 2a (4-hydroxy-3-(thiophen-2-yl)-1,5-dihydro-2H-pyrrol-2-one) or 2b (4-hydroxy-1-methyl3-(thiophen-2-yl)-1,5-dihydro- $2 \mathrm{H}$-pyrrol-2-one) was successfully prepared according a previously procedure [31]. The substituted 2-bromo-1-phenylethan-1-ones $\mathbf{3 a}-\mathbf{3 j}$ were synthesized according to a reported method [32].

\section{General procedures for intermediates 4}

A mixture of a intermediate $2(10 \mathrm{mmol})$, a intermediate $3(11 \mathrm{mmol})$ and triethylamine $(11 \mathrm{mmol})$ in acetone $(50 \mathrm{~mL})$ was stirred at room temperature for $4 \mathrm{~h}$. After that, the white solid appeared in the reaction solution was filtered, washed with water and diethyl ether to obtain a intermediate 4 .

4-(2-oxo-2-(4-methylphenyl)ethoxy)-3-(thiophen-2-yl)-1,5-dihydro-2H-pyrrol-2-one (4a)

Yellow solid, m.p. $179-181{ }^{\circ} \mathrm{C}$, yield $68 \% ;{ }^{1} \mathrm{H}$ NMR $\left(400 \mathrm{MHz}, \mathrm{DMSO}-d_{6}\right) \delta 8.02(\mathrm{~s}, 1 \mathrm{H}$, Pyrroline-1-H), 7.77 $\left(\mathrm{d}, J=7.9 \mathrm{~Hz}, 2 \mathrm{H}, \operatorname{Ar}\left(4-\mathrm{CH}_{3}\right)-2,6-2 \mathrm{H}\right), 7.63(\mathrm{~d}, J=3.0 \mathrm{~Hz}$, $1 \mathrm{H}$, Thiophene-3-H), $7.45(\mathrm{~d}, J=5.0 \mathrm{~Hz}, 1 \mathrm{H}$, Thiophene$5-\mathrm{H}), 7.25\left(\mathrm{~d}, J=7.9 \mathrm{~Hz}, 2 \mathrm{H}, \mathrm{Ar}\left(4-\mathrm{CH}_{3}\right)-3.5-2 \mathrm{H}\right), 6.93(\mathrm{t}$, $J=4.2 \mathrm{~Hz}, 1 \mathrm{H}$, Thiophene-4-H), $5.38\left(\mathrm{~s}, 2 \mathrm{H}, \mathrm{CH}_{2}\right), 4.38$ (s, 2H, Pyrroline-5-2H), $2.32\left(\mathrm{~s}, 3 \mathrm{H}, \mathrm{CH}_{3}\right)$. 
4-(2-oxo-2-phenylethoxy)-3-(thiophen-2-yl)-1,5-dihydro-2H-pyrrol-2-one (4b)

Yellow solid, m.p. $172-174{ }^{\circ} \mathrm{C}$, yield $57 \%$; ${ }^{1} \mathrm{H}$ NMR (400 MHz, DMSO- $\left.d_{6}\right) \delta 7.97$ (s, 1H, Pyrroline-1-H), 7.86 (d, $J=7.8 \mathrm{~Hz}, 2 \mathrm{H}, \mathrm{Ph}-2,6-2 \mathrm{H}), 7.42$ (d, J=3.0 Hz, $1 \mathrm{H}$, Thiophene-3-H), $7.38(\mathrm{~d}, J=5.0 \mathrm{~Hz}, 1 \mathrm{H}$, Thiophene$5-\mathrm{H}), 7.32$ (t, $J=6.7 \mathrm{~Hz}, 2 \mathrm{H}, \mathrm{Ph}-3,5-2 \mathrm{H}), 7.28-7.21(\mathrm{~m}$, $1 \mathrm{H}, \mathrm{Ph}-4-\mathrm{H}), 6.99-6.94(\mathrm{~m}, 1 \mathrm{H}$, Thiophene-4-H), 5.39 (s, 2H, $\mathrm{CH}_{2}$ ), 4.38 (s, 2H, Pyrroline-5-2H).

4-(2-oxo-2-(2-chlorophenyl)ethoxy)-3-(thiophen-2-yl)-1,5-dihydro-2H-pyrrol-2-one (4c)

Yellow solid, m.p. $162-164{ }^{\circ} \mathrm{C}$, yield $57 \%$; ${ }^{1} \mathrm{H}$ NMR (400 MHz, DMSO- $\left.d_{6}\right) \delta 8.05$ (s, 1H, Pyrroline-1-H), $7.62(\mathrm{dd}, J=5.7,3.5 \mathrm{~Hz}, 1 \mathrm{H}, \operatorname{Ar}(2-\mathrm{Cl})-3-\mathrm{H}), 7.56(\mathrm{dt}$, $J=7.3,3.7 \mathrm{~Hz}, 1 \mathrm{H}, \operatorname{Ar}(2-\mathrm{Cl})-4-\mathrm{H}), 7.47$ (dd, $J=5.7$, $3.5 \mathrm{~Hz}, 2 \mathrm{H}$, Thiophene-3,5-2H), $7.28(\mathrm{~d}, J=4.9 \mathrm{~Hz}, 1 \mathrm{H}$, $\operatorname{Ar}(2-\mathrm{Cl})-6-\mathrm{H}), 7.16(\mathrm{~d}, J=5,4 \mathrm{~Hz}, 1 \mathrm{H}$, Thiophene-4-H), 6.87-6.80 (m, 1H, $\operatorname{Ar}(2-\mathrm{Cl})-5-\mathrm{H}), 5.38\left(\mathrm{~s}, 2 \mathrm{H}, \mathrm{CH}_{2}\right)$, 4.27 (s, 2H, Pyrroline-5-2H).

4-(2-oxo-2-(2-bromophenyl)ethoxy)-3-(thiophen-2-yl)-1,5-dihydro-2H-pyrrol-2-one (4d)

Yellow solid, m.p. $152-154{ }^{\circ} \mathrm{C}$, yield $34 \% ;{ }^{1} \mathrm{H}$ NMR (400 MHz, DMSO- $\left.d_{6}\right) \quad \delta 7.93 \quad(\mathrm{~s}, 1 \mathrm{H}$, Pyrroline1-H), 7.68 (d, J=7.8 Hz, 1H, Ar(2-Br)-6-H), 7.55 (d, $J=7.1 \mathrm{~Hz}, 1 \mathrm{H}, \operatorname{Ar}(2-\mathrm{Br})-4-\mathrm{H}), 7.46(\mathrm{t}, J=7.4 \mathrm{~Hz}, 1 \mathrm{H}$, Thiophene-3-H), $7.34(\mathrm{t}, J=7.6 \mathrm{~Hz}, 1 \mathrm{H}$, Thiophene$5-\mathrm{H}), 7.28(\mathrm{~d}, J=4.8 \mathrm{~Hz}, 1 \mathrm{H}$, Thiophene-4-H), 7.11 (d, $J=8.5 \mathrm{~Hz}, 2 \mathrm{H}, \mathrm{Ar}(2-\mathrm{Br})-3,5-2 \mathrm{H}), 5.37\left(\mathrm{~s}, 2 \mathrm{H}, \mathrm{CH}_{2}\right), 4.26$ (s, 2H, Pyrroline-5-2H).

4-(2-oxo-2-(3-chlorophenyl)ethoxy)-3-(thiophen-2-yl)-1,5-dihydro-2H-pyrrol-2-one (4e)

Yellow solid, m.p. $168-170{ }^{\circ} \mathrm{C}$, yield $43 \%$; ${ }^{1} \mathrm{H}$ NMR (400 MHz, DMSO- $\left.d_{6}\right) \delta 7.98$ (s, 1H, Pyrroline-1-H), $7.93(\mathrm{~s}, 1 \mathrm{H}, \operatorname{Ar}(3-\mathrm{Cl})-2-\mathrm{H}), 7.84(\mathrm{~d}, J=7.7 \mathrm{~Hz}, 1 \mathrm{H}$, $\operatorname{Ar}(3-\mathrm{Cl})-6-\mathrm{H}), 7.42(\mathrm{t}, J=8.5 \mathrm{~Hz}, 2 \mathrm{H}$, Thiophene3,5-2H), 7.32 (d, J=4.9 Hz, 1H, Ar(3-Cl)-4-H), 7.23 (d, $J=8.7 \mathrm{~Hz}, 2 \mathrm{H}, \operatorname{Ar}(3-\mathrm{Cl})-5-\mathrm{H}$, Thiophene-4-H), 5.41 (s, $2 \mathrm{H}, \mathrm{CH}_{2}$ ), 4.37 (s, 2H, Pyrroline-5-2H).

4-(2-oxo-2-(4-fluorophenyl)ethoxy)-3-(thiophen-2-yl)-1,5-dihydro-2H-pyrrol-2-one (4f)

Yellow solid, m.p. $174-176{ }^{\circ} \mathrm{C}$, yield $56 \%$; ${ }^{1} \mathrm{H}$ NMR (400 MHz, DMSO- $\left.d_{6}\right) \delta 7.97$ (s, 1H, Pyrroline-1-H), 7.94-7.84 (m, 2H, Ar(4-F)-2,6-2H), 7.41 (d, J=2.6 Hz, $1 \mathrm{H}$, Thiophene-3-H), $7.32(\mathrm{~d}, J=4.8 \mathrm{~Hz}, 1 \mathrm{H}$, Thiophene-5-H), $7.12(\mathrm{t}, J=8.6 \mathrm{~Hz}, 2 \mathrm{H}, \operatorname{Ar}(4-\mathrm{F})-3,5-2 \mathrm{H})$,
6.99-6.85 (m, 1H, Thiophene-4-H), 5.40 (s, 2H, $\mathrm{CH}_{2}$ ), 4.37 (s, 2H, Pyrroline-5-2H).

4-(2-oxo-2-(4-chlorophenyl)ethoxy)-3-(thiophen-2-yl)-1,5-dihydro-2H-pyrrol-2-one (4g)

Yellow solid, m.p. $145-147{ }^{\circ} \mathrm{C}$, yield 91\%; ${ }^{1} \mathrm{H}$ NMR (400 MHz, DMSO- $d_{6}$ ) $\delta 7.99$ (s, $1 \mathrm{H}$, Pyrroline-1-H), 7.89 (d, $J=8.6 \mathrm{~Hz}, 2 \mathrm{H}, \operatorname{Ar}(4-\mathrm{Cl})-2,6-2 \mathrm{H}), 7.40$ (d, $J=3.3 \mathrm{~Hz}$, $1 \mathrm{H}$, Thiophene-3-H), $7.32(\mathrm{~d}, J=4.9 \mathrm{~Hz}, 1 \mathrm{H}$, Thiophene3-H), 7.22 (d, J=8.8 Hz, 2H, Ar(4-Cl)-3,5-2H), 6.93 (dd, $J=8.8,4.8 \mathrm{~Hz}, 1 \mathrm{H}$, Thiophene-4-H), $5.40\left(\mathrm{~s}, 2 \mathrm{H}, \mathrm{CH}_{2}\right)$, 4.37 (s, 2H, Pyrroline-5-2H).

4-(2-oxo-2-(4-bromophenyl)ethoxy)-3-(thiophen-2-yl)-1,5-dihydro-2H-pyrrol-2-one (4h)

Yellow solid, m.p. $156-158{ }^{\circ} \mathrm{C}$, yield $71 \%$; ${ }^{1} \mathrm{H}$ NMR (400 MHz, DMSO- $\left.d_{6}\right) \delta 7.97$ (s, 1H, Pyrroline-1-H), 7.94-7.86 (m, 2H, Ar(4-Br)-2,6-2H), 7.42 (d, J=5.9 Hz, $1 \mathrm{H}$, Thiophene-3-H), $7.32(\mathrm{~d}, J=4.9 \mathrm{~Hz}, 1 \mathrm{H}$, Thiophene5-H), 7.23 (d, J=8.7 Hz, 2H, Ar(4-Br)-3,5-2H), 6.94-6.88 (m, 1H, Thiophene-4- $\mathrm{H}), 5.40\left(\mathrm{~s}, 2 \mathrm{H}, \mathrm{CH}_{2}\right), 4.37(\mathrm{~s}, 2 \mathrm{H}$, Pyrroline-5-2H).

\section{4-(2-oxo-2-(2,4-dichlorophenyl)}

ethoxy)-3-(thiophen-2-yl)-1,5-dihydro-2H-pyrrol-2-one (4i)

Yellow solid, m.p. $152-154{ }^{\circ} \mathrm{C}$, yield $44 \%$; ${ }^{1} \mathrm{H}$ NMR (400 MHz, DMSO- $d_{6}$ ) $\delta 7.95$ (s, $1 \mathrm{H}$, Pyrroline-1-H), 7.68 (d, $J=1.5 \mathrm{~Hz}, 1 \mathrm{H}, \operatorname{Ar}(2,4-2 \mathrm{Cl})-3-\mathrm{H}), 7.61$ (d, $J=8.3 \mathrm{~Hz}$, $1 \mathrm{H}$, Thiophene-3-H), 7.51 (dd, $J=8.3,1.5 \mathrm{~Hz}, 1 \mathrm{H}$, Thiophene-5-H), $7.30(\mathrm{~d}, J=5.1 \mathrm{~Hz}, 1 \mathrm{H}, \operatorname{Ar}(2,4-2 \mathrm{Cl})-5-\mathrm{H})$, $7.11(\mathrm{~d}, J=8.7 \mathrm{~Hz}, 1 \mathrm{H}, \operatorname{Ar}(2,4-2 \mathrm{Cl})-6-\mathrm{H}), \quad 6.90-6.78$ (m, $1 \mathrm{H}$, Thiophene-4- $\mathrm{H}), 5.37\left(\mathrm{~s}, 2 \mathrm{H}, \mathrm{CH}_{2}\right), 4.26(\mathrm{~s}, 2 \mathrm{H}$, Pyrroline-5-2H).

\section{4-(2-oxo-2-(4-methoxyphenyl)}

ethoxy)-3-(thiophen-2-yl)-1,5-dihydro-2H-pyrrol-2-one (4j) Yellow solid, m.p. $156-158{ }^{\circ} \mathrm{C}$, yield $57 \%$; ${ }^{1} \mathrm{H}$ NMR $\left(400 \mathrm{MHz}, \mathrm{DMSO}-d_{6}\right) \delta 7.97(\mathrm{~s}, 1 \mathrm{H}$, Pyrroline-1-H), 7.84-7.76 (m, 2H, $\left.\operatorname{Ar}\left(4-\mathrm{OCH}_{3}\right)-2,6-2 \mathrm{H}\right), \quad 7.42 \quad(\mathrm{~d}$, $J=5.9 \mathrm{~Hz}, 1 \mathrm{H}$, Thiophene-3-H), $7.32(\mathrm{~d}, J=4.9 \mathrm{~Hz}, 1 \mathrm{H}$, Thiophene-5-H), $7.23\left(\mathrm{~d}, J=8.7 \mathrm{~Hz}, 2 \mathrm{H}, \mathrm{Ar}\left(4-\mathrm{OCH}_{3}\right)-\right.$ 3,5-2H), 6.97-6.88 (m, 1H, Thiophene-4-H), $5.40(\mathrm{~s}, 2 \mathrm{H}$, $\mathrm{CH}_{2}$ ), 4.37 (s, 2H, Pyrroline-5-2H), 3.78 (s, 3H, $\mathrm{CH}_{3}$ ).

\section{4-(2-oxo-2-(4-fluorophenyl)ethoxy)-1-me-}

thyl-3-(thiophen-2-yl)-1,5-dihydro-2H-pyrrol-2-one (4k)

Yellow solid, m.p. $166-168{ }^{\circ} \mathrm{C}$, yield $72 \%$; ${ }^{1} \mathrm{H}$ NMR $\left(400 \mathrm{MHz}, \mathrm{DMSO}-d_{6}\right) \delta 7.90$ (dd, $J=8.7,5.6 \mathrm{~Hz}, 2 \mathrm{H}$, $\operatorname{Ar}(4-\mathrm{F})-2,6-2 \mathrm{H}), 7.40(\mathrm{~d}, J=3.6 \mathrm{~Hz}, 1 \mathrm{H}$, Thiophene3-H), 7.32 (d, J=5.1 Hz, 1H, Thiophene-5-H), 7.29 (d, $J=11.1 \mathrm{~Hz}, 2 \mathrm{H}, \operatorname{Ar}(4-\mathrm{F})-3,5-2 \mathrm{H}), 6.94(\mathrm{dd}, J=5.0,3.8 \mathrm{~Hz}$, $1 \mathrm{H}$, Thiophene-4-H), 5.39 (s, 2H, $\left.\mathrm{CH}_{2}\right), 4.45$ (s, 2H, Pyrroline-5-2H), $2.99\left(\mathrm{~s}, 3 \mathrm{H}, \mathrm{CH}_{3}\right)$. 
4-(2-oxo-2-(4-methylphenyl)ethoxy)-1-methyl-3-(thiophen-2-yl)-1,5-dihydro-2H-pyrrol-2-one (4I) Yellow solid, m.p. $143-145{ }^{\circ} \mathrm{C}$, yield $59 \%$; ${ }^{1} \mathrm{H}$ NMR $\left(400 \mathrm{MHz}, \mathrm{DMSO}-d_{6}\right) \delta 7.76(\mathrm{~d}, J=7.7 \mathrm{~Hz}, 2 \mathrm{H}, \operatorname{Ar}(4-$ $\mathrm{CH}_{3}$ )-2,6-2H), 7.41 (d, J=1.8 Hz, $1 \mathrm{H}$, Thiophene-3-H), $7.31\left(\mathrm{~d}, J=8.5 \mathrm{~Hz}, 3 \mathrm{H}\right.$, Thiophene-5-H, $\operatorname{Ar}\left(4-\mathrm{CH}_{3}\right)-$ 3,5-2H), 7.00-6.95 (m, 1H, Thiophene-4-H), 5.37 (s, 2H, $\mathrm{CH}_{2}$ ) 4.45 (s, 2H, Pyrroline-5-2H), 2.99 (s, 3H, $\mathrm{CH}_{3}$ ), $2.32\left(\mathrm{~s}, 3 \mathrm{H}, \mathrm{CH}_{3}\right)$.

\section{General procedures for intermediates 5}

A mixture of a intermediate $4(1.50 \mathrm{mmol})$ and substituted phenylhydrazine $(1.70 \mathrm{mmol})$ in acetonitrile (35 mL) was stirred under $35^{\circ} \mathrm{C}$. After the reaction was completed, the white solid appeared in the reaction solution was filtered and recrystallized with diethyl ether to obtain a title compound $\mathbf{5}$.

\section{(Z)-4-(2-(2-phenylhydrazono)-2-(4-methylphenyl)} ethoxy)-3-(thiophen-2-yl)-1,5-dihydro-2H-pyrrol-2-one (5a) Yellow solid, m.p. $153-155^{\circ} \mathrm{C}$, yield $65 \%$; IR $\left(\mathrm{KBr}, \mathrm{cm}^{-1}\right)$ : 3380, 3171, 3063, 1676; ${ }^{1} \mathrm{H}$ NMR (400 MHz, DMSO- $d_{6}$ ) $\delta 9.98(\mathrm{~s}, 1 \mathrm{H}, \mathrm{Ar}-\mathrm{NH}=\mathrm{N}), 7.97$ (s, 1H, Pyrroline-1-H), $7.78\left(\mathrm{~s}, 1 \mathrm{H}, \operatorname{Ar}\left(4-\mathrm{CH}_{3}\right)-2-\mathrm{H}\right), 7.76\left(\mathrm{~s}, 1 \mathrm{H}, \operatorname{Ar}\left(4-\mathrm{CH}_{3}\right)-\right.$ 6-H), 7.42 (d, J=3.1 Hz, 1H, Thiophene-3-H), 7.33-7.29 $(\mathrm{m}, 1 \mathrm{H}$, Thiophene-5-H), $7.25(\mathrm{t}, J=8.4 \mathrm{~Hz}, 5 \mathrm{H}, \mathrm{Ph}-$ 2,3,5,6-4H, Thiophene-4-H), 7.21 (s, $1 \mathrm{H}, \operatorname{Ar}\left(4-\mathrm{CH}_{3}\right)-$ 3-H), $6.93\left(\mathrm{dd}, J=4.9,3.8 \mathrm{~Hz}, 1 \mathrm{H}, \operatorname{Ar}\left(4-\mathrm{CH}_{3}\right)-5-\mathrm{H}\right)$, $6.83(\mathrm{t}, J=6.5 \mathrm{~Hz}, 1 \mathrm{H}, \mathrm{Ph}-4-\mathrm{H}), 5.40\left(\mathrm{~s}, 2 \mathrm{H}, \mathrm{CH}_{2}\right), 4.38$ (s, 2H, Pyrroline-5-2H), $2.32\left(\mathrm{~s}, 3 \mathrm{H}, \mathrm{CH}_{3}\right) ;{ }^{13} \mathrm{C} \mathrm{NMR}$ $\left(100 \mathrm{MHz}, \mathrm{DMSO}-d_{6}\right) \delta 171.99,167.05,145.68,137.59$, $136.57,134.91,132.66,129.59,129.51,126.77,125.80$, 124.57, 124.04, 120.25, 113.44, 103.76, 61.76, 43.65, 21.28; Anal. Calcd for $\mathrm{C}_{23} \mathrm{H}_{21} \mathrm{~N}_{3} \mathrm{O}_{2} \mathrm{~S}$ (403.14): C, 68.46; H, 5.25; $\mathrm{N}, 10.41$. Found: C, 68.22; H, 5.27; N, 10.37; EI-MS $\mathrm{m} / \mathrm{z}$ 403.14 $[\mathrm{M}]^{+}$.

(Z)-4-(2-(2-(2-fluorophenyl)hydrazono)-2-(4-methylphenyl) ethoxy)-3-(thiophen-2-yl)-1,5-dihydro-2H-pyrrol-2-one (5b) White solid, m.p. $158-160{ }^{\circ} \mathrm{C}$, yield $51 \%$; IR $\left(\mathrm{KBr}, \mathrm{cm}^{-1}\right)$ : 3376, 3177, 3069, 1678; ${ }^{1} \mathrm{H}$ NMR (400 MHz, DMSO- $d_{6}$ ) $\delta$ 9.54 (s, $1 \mathrm{H}, \mathrm{Ar}-\mathrm{NH}=\mathrm{N}), 7.95(\mathrm{~s}, 1 \mathrm{H}$, Pyrroline-1-H), $7.79\left(\mathrm{~d}, J=8.2 \mathrm{~Hz}, 2 \mathrm{H}, \operatorname{Ar}\left(4-\mathrm{CH}_{3}\right)-2,6-2 \mathrm{H}\right), 7.62(\mathrm{td}$, $J=8.5,1.4 \mathrm{~Hz}, 1 \mathrm{H}$, Thiophene-3-H), 7.43-7.39 (m, $1 \mathrm{H}$, Thiophene-5-H), 7.32 (dd, $J=5.1,0.9 \mathrm{~Hz}, 1 \mathrm{H}, \operatorname{Ar}(2-\mathrm{F})-$ 4-H), 7.24 (d, $J=8.1 \mathrm{~Hz}, 2 \mathrm{H}, \operatorname{Ar}(2-\mathrm{F})-3,6-2 \mathrm{H}), 7.21-7.13$ (m, 2H, $\left.\operatorname{Ar}\left(4-\mathrm{CH}_{3}\right)-3,5-2 \mathrm{H}\right), 6.93$ (dd, $J=5.1,3.7 \mathrm{~Hz}, 1 \mathrm{H}$, $\operatorname{Ar}(2-\mathrm{F})-5-\mathrm{H}), 6.91-6.84(\mathrm{~m}, 1 \mathrm{H}$, Thiophene-4-H), 5.51 (s, $2 \mathrm{H}, \mathrm{CH}_{2}$ ), 4.35 (s, 2H, Pyrroline-5-2H), 2.33 (s, 3H, $\mathrm{CH}_{3}$ ); ${ }^{13} \mathrm{C}$ NMR (100 MHz, DMSO-d $) \delta 171.91,166.71,151.54$, $149.15,140.53,138.23,134.40,133.83,133.74,132.52$, $129.53,126.74,126.29,125.52,125.48,124.61,124.07$, 120.75, 120.69, 115.87, 103.82, 62.52, 43.58, 21.30; Anal.
Calcd for $\mathrm{C}_{23} \mathrm{H}_{20} \mathrm{FN}_{3} \mathrm{O}_{2} \mathrm{~S}$ (421.13): C, 65.54; $\mathrm{H}, 4.78 ; \mathrm{N}$, 9.97. Found: C, 65.12; H, 4.81; N, 9.92; EI-MS $m / z 421.13$ $[\mathrm{M}]^{+}$.

\section{(Z)-4-(2-(2-(2-chlorophenyl)hydrazono)-2-(4-methylphenyl)} ethoxy)-3-(thiophen-2-yl)-1,5-dihydro-2H-pyrrol-2-one (5c) White solid, m.p. $160-162{ }^{\circ} \mathrm{C}$, yield $30 \%$; IR $\left(\mathrm{KBr}, \mathrm{cm}^{-1}\right)$ : 3376, 3176, 3070, 1679; ${ }^{1} \mathrm{H}$ NMR (400 MHz, DMSO- $d_{6}$ ) $\delta 9.12(\mathrm{~s}, 1 \mathrm{H}, \mathrm{Ar}-\mathrm{NH}=\mathrm{N}), 7.99(\mathrm{~s}, 1 \mathrm{H}$, Pyrroline-1-H), $7.82\left(\mathrm{~d}, J=8.2 \mathrm{~Hz}, 2 \mathrm{H}, \operatorname{Ar}\left(4-\mathrm{CH}_{3}\right)-2,6-2 \mathrm{H}\right), 7.64(\mathrm{~d}$, $J=7.2 \mathrm{~Hz}, 1 \mathrm{H}$, Thiophene-3-H), $7.48(\mathrm{~d}, J=2.9 \mathrm{~Hz}, 1 \mathrm{H}$, Thiophene-5-H), 7.36 (d, J=4.1 Hz, $1 \mathrm{H}, \operatorname{Ar}(2-\mathrm{Cl})-3-\mathrm{H})$, 7.35-7.30 (m, 2H, Thiophene-4-H, $\mathrm{Ar}(2-\mathrm{Cl})-5-\mathrm{H}), 7.26$ $\left(\mathrm{d}, J=8.1 \mathrm{~Hz}, 2 \mathrm{H}, \operatorname{Ar}\left(4-\mathrm{CH}_{3}\right)-3,5-2 \mathrm{H}\right), 6.96(\mathrm{dd}, J=5.0$, $3.7 \mathrm{~Hz}, 1 \mathrm{H}, \operatorname{Ar}(2-\mathrm{Cl})-6-\mathrm{H}), 6.92-6.86(\mathrm{~m}, 1 \mathrm{H}, \operatorname{Ar}(2-\mathrm{Cl})-$ 4-H), 5.58 (s, 2H, $\mathrm{CH}_{2}$ ), 4.35 (s, 2H, Pyrroline-5-2H), 2.34 $\left(\mathrm{s}, 3 \mathrm{H}, \mathrm{CH}_{3}\right) ;{ }^{13} \mathrm{C}$ NMR $\left(100 \mathrm{MHz}, \mathrm{DMSO}-d_{6}\right) \delta 171.74$, $165.99,141.52,141.27,138.68,133.97,132.25,129.80$, $129.67,128.69,126.76,126.33,124.85,124.45,121.55$, 118.12, 115.32, 104.37, 63.53, 43.56, 21.31; Anal. Calcd for $\mathrm{C}_{23} \mathrm{H}_{20} \mathrm{ClN}_{3} \mathrm{O}_{2} \mathrm{~S}$ (437.1): C, 63.08; $\mathrm{H}, 4.60 ; \mathrm{N}, 9.60$. Found: C, 62.82; H, 4.62; N, 9.57; EI-MS $m / z 437.1\left[\mathrm{M}^{+}\right.$.

\section{(Z)-4-(2-(2-(3-chlorophenyl)hydrazono)-2-(4-methylphenyl)} ethoxy)-3-(thiophen-2-yl)-1,5-dihydro-2H-pyrrol-2-one (5d) White solid, m.p. $172-174{ }^{\circ} \mathrm{C}$, yield $38 \%$; IR $\left(\mathrm{KBr}, \mathrm{cm}^{-1}\right)$ : 3376, 3192, 3069, 1676; ${ }^{1} \mathrm{H}$ NMR (400 MHz, DMSO- $d_{6}$ ) $\delta 10.12(\mathrm{~s}, 1 \mathrm{H}, \mathrm{Ar}-\mathrm{NH}=\mathrm{N}), 7.97$ (s, 1H, Pyrroline-1-H), $7.77\left(\mathrm{~d}, J=8.2 \mathrm{~Hz}, 2 \mathrm{H}, \operatorname{Ar}\left(4-\mathrm{CH}_{3}\right)-2,6-2 \mathrm{H}\right), 7.41(\mathrm{~d}$, $J=2.8 \mathrm{~Hz}, 1 \mathrm{H}, \operatorname{Ar}(3-\mathrm{Cl})-3-\mathrm{H}), 7.34-7.30$ (m, $1 \mathrm{H}$, Thiophene-3-H), $7.27(\mathrm{t}, J=5.2 \mathrm{~Hz}, 2 \mathrm{H}$, Thiophene-4,5-2H), 7.25 (s, 1H, Ar(3-Cl)-5-H), 7.23 (s, 1H, Ar(3-Cl)-4-H), $7.18\left(\mathrm{~d}, J=8.2 \mathrm{~Hz}, 1 \mathrm{H}, \operatorname{Ar}\left(4-\mathrm{CH}_{3}\right)-3-\mathrm{H}\right), 6.93$ (dd, $J=5.0$, $\left.3.7 \mathrm{~Hz}, 1 \mathrm{H}, \operatorname{Ar}\left(4-\mathrm{CH}_{3}\right)-5-\mathrm{H}\right), 6.85$ (dd, $J=7.8,1.1 \mathrm{~Hz}, 1 \mathrm{H}$, $\mathrm{Ar}(3-\mathrm{Cl})-6-\mathrm{H}), 5.39$ (s, 2H, $\mathrm{CH}_{2}$ ), 4.37 (s, 2H, Pyrroline5-2H), 2.33 (s, 3H, $\left.\mathrm{CH}_{3}\right) ;{ }^{13} \mathrm{C}$ NMR (100 MHz, DMSO-d $)$ $\delta$ 171.94, 166.89, 147.15, 138.39, 138.06, 134.53, 134.24, $132.60,131.27,129.57,126.76,126.05,124.63,124.07$, 119.64, 112.79, 112.07, 103.83, 61.84, 43.63, 21.30; Anal. Calcd for $\mathrm{C}_{23} \mathrm{H}_{20} \mathrm{ClN}_{3} \mathrm{O}_{2} \mathrm{~S}$ (437.1): C, 63.08; H, 4.60; N, 9.60. Found: C, 62.81; H, 4.64; N, 9.66; EI-MS $m / z 437.1$ $[\mathrm{M}]^{+}$.

(Z)-4-(2-(2-(4-fluorophenyl)hydrazono)-2-(4-methylphenyl) ethoxy)-3-(thiophen-2-yl)-1,5-dihydro-2H-pyrrol-2-one (5e) White solid, m.p. $149-151{ }^{\circ} \mathrm{C}$, yield $63 \%$; IR $\left(\mathrm{KBr}, \mathrm{cm}^{-1}\right)$ : 3368, 3167, 3063, 1676; ${ }^{1} \mathrm{H}$ NMR (400 MHz, DMSO- $d_{6}$ ) $\delta 10.05(\mathrm{~s}, 1 \mathrm{H}, \mathrm{Ar}-\mathrm{NH}=\mathrm{N}), 7.99$ (s, 1H, Pyrroline-1-H), $7.76\left(\mathrm{~d}, J=8.0 \mathrm{~Hz}, 2 \mathrm{H}, \operatorname{Ar}\left(4-\mathrm{CH}_{3}\right)-2,6-2 \mathrm{H}\right), 7.41(\mathrm{~d}$, $J=3.3 \mathrm{~Hz}, 1 \mathrm{H}$, Thiophene-3-H), $7.32(\mathrm{~d}, J=5.0 \mathrm{~Hz}, 1 \mathrm{H}$, Thiophene-5-H), 7.25 (dd, $J=10.0,6.3 \mathrm{~Hz}, 3 \mathrm{H}$, Thiophene-4-H, $\operatorname{Ar}(4-\mathrm{F})-3,5-2 \mathrm{H}), 7.21$ (s, $1 \mathrm{H}, \operatorname{Ar}\left(4-\mathrm{CH}_{3}\right)-$ $3-\mathrm{H}), 7.12(\mathrm{t}, J=8.7 \mathrm{~Hz}, 2 \mathrm{H}, \operatorname{Ar}(4-\mathrm{F})-2,6-2 \mathrm{H}), 6.95-6.90$ 
(m, 1H, $\left.\operatorname{Ar}\left(4-\mathrm{CH}_{3}\right)-5-\mathrm{H}\right), 5.40\left(\mathrm{~s}, 2 \mathrm{H}, \mathrm{CH}_{2}\right), 4.39$ (s, $2 \mathrm{H}$, Pyrroline-5-2H), 2.32 (s, 3H, $\left.\mathrm{CH}_{3}\right) ;{ }^{13} \mathrm{C}$ NMR $(100 \mathrm{MHz}$, DMSO- $\left.d_{6}\right) \delta 171.97,167.03,158.07,155.74,142.37$, $137.59,136.71,134.83,132.65,129.49,126.76,125.81$, $124.56,124.02,116.20,115.98,114.53,114.46,103.74$, 61.86, 43.66, 21.27; Anal. Calcd for $\mathrm{C}_{23} \mathrm{H}_{20} \mathrm{FN}_{3} \mathrm{O}_{2} \mathrm{~S}$ (421.1): C, 65.54; H, 4.78; N, 9.97. Found: C, 65.81; H, 4.82; N, 9.89; EI-MS $m / z 421.1[\mathrm{M}]^{+}$.

\section{(Z)-4-(2-(2-(4-chlorophenyl)hydrazono)-2-(4-methylphenyl)} ethoxy)-3-(thiophen-2-yl)-1,5-dihydro-2H-pyrrol-2-one (5f) White solid, m.p. $156-157^{\circ} \mathrm{C}$, yield $61 \%$; IR $\left(\mathrm{KBr}, \mathrm{cm}^{-1}\right)$ : 3366, 3173, 3071, 1677; ${ }^{1} \mathrm{H}$ NMR (400 MHz, DMSO- $d_{6}$ ) $\delta 10.09$ (s, 1H, Ar-NH=N), 7.97 (s, 1H, Pyrroline-1-H), $7.77\left(\mathrm{~d}, J=7.9 \mathrm{~Hz}, 2 \mathrm{H}, \operatorname{Ar}\left(4-\mathrm{CH}_{3}\right)-2,6-2 \mathrm{H}\right), 7.41$ (d, $J=2.7 \mathrm{~Hz}, 1 \mathrm{H}$, Thiophene-3-H), 7.31 (d, $J=8.8 \mathrm{~Hz}, 3 \mathrm{H}$, Thiophene-5-H, $\operatorname{Ar}(4-\mathrm{Cl})-3,5-2 \mathrm{H}), 7.25$ (d, J=10.3 Hz, $3 \mathrm{H}$, Thiophene-4- $\mathrm{H}, \operatorname{Ar}(4-\mathrm{Cl})-2,6-2 \mathrm{H}), 7.21$ (s, 1H, $\operatorname{Ar}(4-$ $\left.\left.\mathrm{CH}_{3}\right)-3-\mathrm{H}\right), 6.96-6.90\left(\mathrm{~m}, 1 \mathrm{H}, \operatorname{Ar}\left(4-\mathrm{CH}_{3}\right)-5-\mathrm{H}\right), 5.39$ (s, $2 \mathrm{H}, \mathrm{CH}_{2}$ ), 4.37 (s, 2H, Pyrroline-5-2H), $2.32\left(\mathrm{~s}, 3 \mathrm{H}, \mathrm{CH}_{3}\right.$ ); ${ }^{13} \mathrm{C}$ NMR $\left(100 \mathrm{MHz}, \mathrm{DMSO}-d_{6}\right) \delta 171.96,166.93,144.64$, $137.86,137.60,134.66,132.62,129.52,129.41,126.77$, $125.93,124.60,124.06,123.57,114.90,103.82,61.81$, 43.64, 21.29; Anal. Calcd for $\mathrm{C}_{23} \mathrm{H}_{20} \mathrm{ClN}_{3} \mathrm{O}_{2} \mathrm{~S}$ (437.1): C, 63.08; H, 4.60; N, 9.60. Found: C, 63.51; H, 4.64; N, 9.67; EI-MS $m / z 437.1[\mathrm{M}]^{+}$.

(Z)-4-(2-(2-(4-bromophenyl)hydrazono)-2-(4-methylphenyl) ethoxy)-3-(thiophen-2-yl)-1,5-dihydro-2H-pyrrol-2-one (5g) White solid, m.p. $160-162{ }^{\circ} \mathrm{C}$, yield $72 \%$; IR $\left(\mathrm{KBr}, \mathrm{cm}^{-1}\right)$ : 3364, 3179, 3075, 1677; ${ }^{1} \mathrm{H}$ NMR (400 MHz, DMSO-d $)$ $\delta 10.09$ (s, 1H, Ar-NH=N), 7.97 (s, 1H, Pyrroline-1-H), $7.77\left(\mathrm{~d}, J=8.1 \mathrm{~Hz}, 2 \mathrm{H}, \operatorname{Ar}\left(4-\mathrm{CH}_{3}\right)-2,6-2 \mathrm{H}\right), 7.42(\mathrm{~d}$, $J=8.7 \mathrm{~Hz}, 3 \mathrm{H}$, Thiophene-3,5-2H, Ar(4-Br)-3-H), 7.31 $(\mathrm{d}, J=5.0 \mathrm{~Hz}, 1 \mathrm{H}, \operatorname{Ar}(4-\mathrm{Br})-5-\mathrm{H}), 7.21(\mathrm{t}, J=8.1 \mathrm{~Hz}, 4 \mathrm{H}$, Thiophene-4- $\left.\mathrm{H}, \mathrm{Ar}\left(4-\mathrm{CH}_{3}\right)-3,5-2 \mathrm{H}, \mathrm{Ar}(4-\mathrm{Br})-2-\mathrm{H}\right), 6.96-$ 6.90 (m, 1H, Ar(4-Br)-6-H), 5.38 (s, 2H, CH $\left.\mathrm{CH}_{2}\right), 4.37$ (s, 2H, Pyrroline-5-2H), 2.32 (s, 3H, $\left.\mathrm{CH}_{3}\right) ;{ }^{13} \mathrm{C}$ NMR (100 MHz, DMSO- $\left.d_{6}\right) \delta 171.95,166.92,145.02,137.89,137.70$, $134.65,132.62,132.25,129.53,126.77,125.94,124.61$, 124.06, 115.40, 111.25, 103.82, 61.82, 43.63, 21.29; Anal. Calcd for $\mathrm{C}_{23} \mathrm{H}_{20} \mathrm{BrN}_{3} \mathrm{O}_{2} \mathrm{~S}$ (481.0): C, 57.27; H, 4.18; N, 8.71. Found: C, 57.14; H, 4.21; N, 8.72; EI-MS $m / z 481.0$ $[\mathrm{M}]^{+}$.

\section{(Z)-4-(2-(2-(2-(4-(trifluoromethyl)phe-}

\section{nyl)hydrazono)-2-(4-methylphenyl)}

\section{ethoxy)-3-(thiophen-2-yl)-1,5-dihydro-2H-pyrrol-2-one (5h)}

White solid, m.p. $167-169^{\circ} \mathrm{C}$, yield $82 \%$; IR $\left(\mathrm{KBr}, \mathrm{cm}^{-1}\right)$ : 3363, 3172, 3074, 1681, 1590; ${ }^{1} \mathrm{H}$ NMR (400 MHz, DMSO-d 6 ) $\delta 10.35(\mathrm{~s}, 1 \mathrm{H}, \mathrm{Ar}-\mathrm{NH}=\mathrm{N}), 7.98(\mathrm{~s}, 1 \mathrm{H}, \mathrm{Pyr}-$ roline-1-H), $7.80\left(\mathrm{~d}, J=7.7 \mathrm{~Hz}, 2 \mathrm{H}, \mathrm{Ar}\left(4-\mathrm{CF}_{3}\right)-3,5-2 \mathrm{H}\right)$, $7.61\left(\mathrm{~d}, J=8.3 \mathrm{~Hz}, 2 \mathrm{H}, \operatorname{Ar}\left(4-\mathrm{CH}_{3}\right)-2,6-2 \mathrm{H}\right), 7.40$ (s, 2H,
Thiophene-3,5-2H), 7.38 (s, $\left.1 \mathrm{H}, \operatorname{Ar}\left(4-\mathrm{CF}_{3}\right)-2-\mathrm{H}\right), 7.31$ $\left(\mathrm{d}, J=4.9 \mathrm{~Hz}, 1 \mathrm{H}, \operatorname{Ar}\left(4-\mathrm{CF}_{3}\right)-6-\mathrm{H}\right), 7.24(\mathrm{~d}, J=7.7 \mathrm{~Hz}$, 2H, $\left.\operatorname{Ar}\left(4-\mathrm{CH}_{3}\right)-3,5-2 \mathrm{H}\right), 6.92(\mathrm{~d}, J=3.7 \mathrm{~Hz}, 1 \mathrm{H}$, Thiophene-4-H), $5.42\left(\mathrm{~s}, 2 \mathrm{H}, \mathrm{CH}_{2}\right), 4.38$ (s, 2H, Pyrroline-5-2H), 2.34 (s, 3H, $\left.\mathrm{CH}_{3}\right) ;{ }^{13} \mathrm{C}$ NMR (100 MHz, DMSO- $\left.d_{6}\right) \delta 171.94,166.83,148.76,139.34,138.29$, 134.42 , 132.59, 129.56, 126.98, 126.94, 126.76, 126.20, 124.64, 124.07, 120.13, 119.82, 113.23, 103.87, 61.87, 43.63, 21.30; Anal. Calcd for $\mathrm{C}_{24} \mathrm{H}_{20} \mathrm{~F}_{3} \mathrm{~N}_{3} \mathrm{O}_{2} \mathrm{~S}$ (471.1): C, 61.14; H, 4.28; N, 8.91. Found: C, 61.21; H, 4.31; N, 8.89; EI-MS $m / z 471.1[\mathrm{M}]^{+}$.

(Z)-4-(2-(2-(2-(2,4-dichlorophenyl)hydrazono)-2-(4-methylphenyl)ethoxy)-3-(thiophen-2-yl)-1,5-dihydro-2H-pyrrol-2-one (5i)

White solid, m.p. $172-174{ }^{\circ} \mathrm{C}$, yield $39 \%$; IR (KBr, $\left.\mathrm{cm}^{-1}\right)$ : 3363, 3167, 3075, 1679; ${ }^{1} \mathrm{H}$ NMR (400 MHz, DMSO- $\left.d_{6}\right) \delta 9.20(\mathrm{~s}, 1 \mathrm{H}, \mathrm{Ar}-\mathrm{NH}=\mathrm{N}), 8.00$ (s, 1H, Pyrroline-1-H), $7.82\left(\mathrm{~d}, J=7.9 \mathrm{~Hz}, 2 \mathrm{H}, \mathrm{Ar}\left(4-\mathrm{CH}_{3}\right)-2,6-2 \mathrm{H}\right)$, $7.64(\mathrm{~d}, J=8.9 \mathrm{~Hz}, 1 \mathrm{H}$, Thiophene-3-H), $7.53(\mathrm{~s}, 1 \mathrm{H}$, Thiophene-6-H), $7.46(\mathrm{~d}, J=3.0 \mathrm{~Hz}, 1 \mathrm{H}$, Thiophene4-H), 7.39 (d, J=8.8 Hz, 1H, $\operatorname{Ar}(2,4-2 \mathrm{Cl})-6-\mathrm{H}), 7.35$ (d, $J=5.0 \mathrm{~Hz}, 1 \mathrm{H}, \operatorname{Ar}(2,4-2 \mathrm{Cl})-3-\mathrm{H}), 7.26(\mathrm{~d}, J=7.9 \mathrm{~Hz}$, $\left.2 \mathrm{H}, \operatorname{Ar}\left(4-\mathrm{CH}_{3}\right)-3,5-2 \mathrm{H}\right), 6.98-6.93(\mathrm{~m}, 1 \mathrm{H}, \operatorname{Ar}(2,4-2 \mathrm{Cl})-$ 5-H), $5.58\left(\mathrm{~s}, 2 \mathrm{H}, \mathrm{CH}_{2}\right), 4.35$ (s, 2H, Pyrroline-5-2H), $2.34\left(\mathrm{~s}, 3 \mathrm{H}, \mathrm{CH}_{3}\right) ;{ }^{13} \mathrm{C} \mathrm{NMR}\left(100 \mathrm{MHz}, \mathrm{DMSO}-d_{6}\right) \delta$ $171.73,165.98,142.51,140.59,138.88,133.78,132.24$, $129.66,129.09,128.71,126.75,126.46,124.84,124.43$, 124.26, 118.71, 116.46, 104.34, 63.57, 43.55, 21.32; Anal. Calcd for $\mathrm{C}_{23} \mathrm{H}_{19} \mathrm{Cl}_{2} \mathrm{~N}_{3} \mathrm{O}_{2} \mathrm{~S}$ (471.1): C, 58.48; $\mathrm{H}, 4.05 ; \mathrm{N}$, 8.90. Found: C, 58.23; H, 4.21; N, 8.86; EI-MS $m / z 471.1$ $[\mathrm{M}]^{+}$.

(Z)-4-(2-(2-(2-(4-methylphenyl)hydrazono)-2-(4-methylphenyl)ethoxy)-3-(thiophen-2-yl)-1,5-dihydro-2H-pyrrol-2-one (5j) White solid, m.p. $141-143{ }^{\circ} \mathrm{C}$, yield $42 \%$; IR $\left(\mathrm{KBr}, \mathrm{cm}^{-1}\right)$ : 3376, 3172, 3069, 1667; ${ }^{1} \mathrm{H}$ NMR (400 MHz, DMSO- $\left.d_{6}\right) \delta$ $9.90(\mathrm{~s}, 1 \mathrm{H}, \mathrm{Ar}-\mathrm{NH}=\mathrm{N}), 7.98$ (s, 1H, Pyrroline-1-H), 7.75 $\left(\mathrm{d}, J=7.9 \mathrm{~Hz}, 2 \mathrm{H}, \operatorname{Ar}\left(4-\mathrm{CH}_{3}\right)-2,6-2 \mathrm{H}\right), 7.42(\mathrm{~d}, J=3.0 \mathrm{~Hz}$, $1 \mathrm{H}$, Thiophene-3-H), $7.31(\mathrm{~d}, J=5.0 \mathrm{~Hz}, 1 \mathrm{H}$, Thiophene5-H), 7.21 (d, J=7.9 Hz, 2H, $\left.\operatorname{Ar}\left(4-\mathrm{CH}_{3}\right)-3.5-2 \mathrm{H}\right), 7.11$ (dd, $\left.J=27.3,8.1 \mathrm{~Hz}, 4 \mathrm{H}, \operatorname{Ar}\left(4-\mathrm{CH}_{3}\right)-2,3,4,5-4 \mathrm{H}\right), 6.93(\mathrm{t}$, $J=4.2 \mathrm{~Hz}, 1 \mathrm{H}$, Thiophene-4-H), $5.38\left(\mathrm{~s}, 2 \mathrm{H}, \mathrm{CH}_{2}\right), 4.38$ (s, 2H, Pyrroline-5-2H), $2.32\left(\mathrm{~s}, 3 \mathrm{H}, \mathrm{CH}_{3}\right), 2.23(\mathrm{~s}, 3 \mathrm{H}$, $\left.\mathrm{CH}_{3}\right) ;{ }^{13} \mathrm{C}$ NMR (100 MHz, DMSO- $\left.d_{6}\right) \delta 171.98,167.09$, 143.42 , 137.39, 135.82, 135.01, 132.67, 130.02, 129.49, $128.85,126.76,125.66,124.55,124.02,113.44,103.72$, 61.73, 43.64, 21.27, 20.75; Anal. Calcd for $\mathrm{C}_{24} \mathrm{H}_{23} \mathrm{~N}_{3} \mathrm{O}_{2} \mathrm{~S}$ (417.1): C, 58.48; H, 4.05; N, 8.90. Found: C, 58.23; H, 4.07; N, 8.86; EI-MS $m / z 417.1\left[\mathrm{M}^{+}\right.$. 
(Z)-4-(2-(2-(2-(4-methoxyphenyl)hydrazono)-2-(4-methylphenyl)ethoxy)-3-(thiophen-2-yl)-1,5-dihydro-2H-pyrrol-2-one (5k)

White solid, m.p. $140-142{ }^{\circ} \mathrm{C}$, yield $38 \%$; IR $\left(\mathrm{KBr}, \mathrm{cm}^{-1}\right)$ : 3376, 3177, 3069, 1679; ${ }^{1} \mathrm{H}$ NMR (400 MHz, DMSO- $d_{6}$ ) $\delta 9.82(\mathrm{~s}, 1 \mathrm{H}, \mathrm{Ar}-\mathrm{NH}=\mathrm{N}), 7.96(\mathrm{~s}, 1 \mathrm{H}$, Pyrroline-1-H), $7.74\left(\mathrm{~d}, J=8.2 \mathrm{~Hz}, 2 \mathrm{H}, \operatorname{Ar}\left(4-\mathrm{CH}_{3}\right)-2,6-2 \mathrm{H}\right), 7.42(\mathrm{~d}$, $J=3.4 \mathrm{~Hz}, 1 \mathrm{H}$, Thiophene-3-H), $7.32(\mathrm{~d}, J=5.0 \mathrm{~Hz}, 1 \mathrm{H}$, Thiophene-5-H), $7.19(\mathrm{t}, J=9.0 \mathrm{~Hz}, 4 \mathrm{H}$, Thiophene-4- $\mathrm{H}$, $\left.\operatorname{Ar}\left(4-\mathrm{OCH}_{3}\right)-2,6-2 \mathrm{H}, \mathrm{Ar}\left(4-\mathrm{CH}_{3}\right)-3-\mathrm{H}\right), 6.96-6.92(\mathrm{~m}, 1 \mathrm{H}$, $\left.\operatorname{Ar}\left(4-\mathrm{CH}_{3}\right)-5-\mathrm{H}\right), 6.89\left(\mathrm{~d}, J=9.0 \mathrm{~Hz}, 2 \mathrm{H}, \operatorname{Ar}\left(4-\mathrm{OCH}_{3}\right)-\right.$ 3,5-2H), 5.37 (s, 2H, $\mathrm{CH}_{2}$ ), 4.37 (s, 2H, Pyrroline-5-2H), 3.70 (s, 3H, $\left.\mathrm{CH}_{3}\right), 2.31$ (s, 3H, $\left.\mathrm{CH}_{3}\right) ;{ }^{13} \mathrm{C} \mathrm{NMR} \mathrm{(100} \mathrm{MHz,}$ DMSO- $\left.d_{6}\right) \delta 172.01,167.12,153.75,139.63,137.22$, $135.28,135.10,132.69,129.48,126.76,125.56,124.54$, 124.03, 115.02, 114.50, 103.73, 61.75, 55.70, 43.65, 21.25; Anal. Calcd for $\mathrm{C}_{24} \mathrm{H}_{23} \mathrm{~N}_{3} \mathrm{O}_{3} \mathrm{~S}$ (433.1): C, 66.49; $\mathrm{H}, 5.35$; $\mathrm{N}, 9.69$. Found: C, 66.26; H, 5.33; N, 9.73; EI-MS $\mathrm{m} / \mathrm{z}$ $433.1[\mathrm{M}]^{+}$.

(Z)-4-(2-(2-(2-(4-fluorophenyl)hydrazono)-2-phenylethoxy)-3 -(thiophen-2-yl)-1,5-dihydro-2H-pyrrol-2-one (5I)

White solid, m.p. $131-133{ }^{\circ} \mathrm{C}$, yield $44 \%$; IR $\left(\mathrm{KBr}, \mathrm{cm}^{-1}\right)$ : 3343, 3231, 3060, 1677; ${ }^{1} \mathrm{H}$ NMR (400 MHz, DMSO-d $)$ $\delta 10.05(\mathrm{~s}, 1 \mathrm{H}, \mathrm{Ar}-\mathrm{NH}=\mathrm{N}), 7.97$ (s, 1H, Pyrroline-1-H), 7.86 (d, $J=7.8 \mathrm{~Hz}, 2 \mathrm{H}, \mathrm{Ph}-2,6-2 \mathrm{H}), 7.40$ (d, $J=7.5 \mathrm{~Hz}, 3 \mathrm{H}$, Thiophene-3,4,5-3H), $7.32(\mathrm{t}, J=6.7 \mathrm{~Hz}, 2 \mathrm{H}, \mathrm{Ph}-3,5-2 \mathrm{H})$, 7.28-7.21 (m, 2H, $\operatorname{Ar}(4-\mathrm{F})-2,6-2 \mathrm{H}), 7.12(\mathrm{t}, J=8.7 \mathrm{~Hz}$, 2H, Ar(4-F)-3,5-2H), 6.95-6.90 (m, 1H, Ph-4-H), 5.40 (s, 2H, $\mathrm{CH}_{2}$ ), 4.38 (s, 2H, Pyrroline-5-2H); ${ }^{13} \mathrm{C}$ NMR $\left(100 \mathrm{MHz}, \mathrm{DMSO}-d_{6}\right) \delta 171.97,166.96,158.19,155.85$, $142.23,137.58,136.62,132.63,128.89,128.20,126.76$, $125.88,124.59,124.04,116.26,116.04,114.64,114.56$, 103.81, 61.81, 43.64; Anal. Calcd for $\mathrm{C}_{22} \mathrm{H}_{18} \mathrm{FN}_{3} \mathrm{O}_{2} \mathrm{~S}$ (407.1): C, 64.85; H, 4.45; N, 10.31. Found: C, 64.78; H, 4.48; N, 10.37; EI-MS $m / z$ 407.1 [M] ${ }^{+}$.

(Z)-4-(2-(2-chlorophenyl)-2-(2-(4-fluorophenyl)hydrazono) ethoxy)-3-(thiophen-2-yl)-1,5-dihydro-2H-pyrrol-2-one (5m) Yellow solid, m.p. $125-127{ }^{\circ} \mathrm{C}$, yield $46 \%$; IR $\left(\mathrm{KBr}, \mathrm{cm}^{-1}\right)$ : 3312, 3223, 3084, 1682; ${ }^{1} \mathrm{H}$ NMR (400 MHz, DMSO- $\left.d_{6}\right) \delta$ 9.85 (s, 1H, Ar-NH=N), 7.95 (s, 1H, Pyrroline-1-H), 7.58 (dd, $J=5.7,3.5 \mathrm{~Hz}, 1 \mathrm{H}, \operatorname{Ar}(2-\mathrm{Cl})-3-\mathrm{H}), 7.50$ (dt, $J=7.3$, $3.7 \mathrm{~Hz}, 1 \mathrm{H}, \mathrm{Ar}(2-\mathrm{Cl})-4-\mathrm{H}), 7.42(\mathrm{dd}, J=5.7,3.5 \mathrm{~Hz}, 2 \mathrm{H}$, Thiophene-3,5-2H), $7.28(\mathrm{~d}, J=4.9 \mathrm{~Hz}, 1 \mathrm{H}$, Thiophene4-H), 7.20-7.06 (m, 4H, Ar(4-F)-2,3,6-3H, $\operatorname{Ar}(2-\mathrm{Cl})-6-\mathrm{H})$, 7.04 (d, $J=3.1 \mathrm{~Hz}, 1 \mathrm{H}, \operatorname{Ar}(4-\mathrm{F})-5-\mathrm{H}), 6.87-6.80(\mathrm{~m}, 1 \mathrm{H}$, $\operatorname{Ar}(2-\mathrm{Cl})-5-\mathrm{H}), 5.38$ (s, 2H, $\mathrm{CH}_{2}$ ), 4.27 (s, 2H, Pyrroline$5-2 \mathrm{H}) ;{ }^{13} \mathrm{C}$ NMR $\left(100 \mathrm{MHz}, \mathrm{DMSO}-d_{6}\right) \delta 171.85,166.09$, $158.18,155.85,142.37,139.22,136.89,132.68,132.34$, $131.70,130.14,129.95,127.66,126.58,124.56,124.09$, 116.15, 115.93, 114.64, 114.57, 103.88, 65.93, 43.54; Anal. Calcd for $\mathrm{C}_{22} \mathrm{H}_{17} \mathrm{FClN}_{3} \mathrm{O}_{2} \mathrm{~S}$ (441.1): C, 59.80; H, 3.88; N,
9.51. Found: C, 59.78; H, 3.90; N, 9.57; EI-MS $m / z 441.1$ $[\mathrm{M}]^{+}$.

(Z)-4-(2-(2-bromophenyl)-2-(2-(4-fluorophenyl)hydrazono) ethoxy)-3-(thiophen-2-yl)-1,5-dihydro-2H-pyrrol-2-one (5n) Yellow solid, m.p. $132-134{ }^{\circ} \mathrm{C}$, yield $35 \%$; IR $\left(\mathrm{KBr}, \mathrm{cm}^{-1}\right)$ : 3315, 3219, 3087, 1681; ${ }^{1} \mathrm{H}$ NMR (400 MHz, DMSO- $d_{6}$ ) $\delta 9.94(\mathrm{~s}, 1 \mathrm{H}, \mathrm{Ar}-\mathrm{NH}=\mathrm{N}), 7.96(\mathrm{~s}, 1 \mathrm{H}$, Pyrroline-1-H), 7.68 (d, $J=7.9 \mathrm{~Hz}, 1 \mathrm{H}, \operatorname{Ar}(2-\mathrm{Br})-3-\mathrm{H}), 7.55$ (d, $J=6.2 \mathrm{~Hz}$, $1 \mathrm{H}, \operatorname{Ar}(2-\mathrm{Br})-4-\mathrm{H}), 7.49-7.31(\mathrm{~m}, 4 \mathrm{H}$, Thiophene-3,5-2H, $\operatorname{Ar}(4-\mathrm{F})-2,6-2 \mathrm{H}), 7.28(\mathrm{~d}, J=4.9 \mathrm{~Hz}, 1 \mathrm{H}$, Thiophene4-H), $7.11(\mathrm{~d}, J=8.8 \mathrm{~Hz}, 2 \mathrm{H}, \operatorname{Ar}(4-\mathrm{F})-3,5-2 \mathrm{H}), 7.00(\mathrm{~d}$, $J=3.2 \mathrm{~Hz}, 1 \mathrm{H}, \operatorname{Ar}(2-\mathrm{Br})-6-\mathrm{H}), 6.86-6.79(\mathrm{~m}, 1 \mathrm{H}, \operatorname{Ar}(2-$ $\mathrm{Br})-5-\mathrm{H}), 5.37$ (s, 2H, $\left.\mathrm{CH}_{2}\right), 4.26(\mathrm{~s}, 2 \mathrm{H}$, Pyrroline-5-2H); ${ }^{13} \mathrm{C}$ NMR $\left(100 \mathrm{MHz}, \mathrm{DMSO}-d_{6}\right) \delta 171.86,166.06,142.42$, $140.36,138.78,133.08,132.32,131.85,130.31,128.11$, $126.58,124.55,124.16,122.75,116.95,116.87,116.28$, $116.12,116.05,115.90,114.63,114.56,103.90,66.02$, 43.62; Anal. Calcd for $\mathrm{C}_{22} \mathrm{H}_{17} \mathrm{FBrN}_{3} \mathrm{O}_{2} \mathrm{~S}$ (485.0): C, 54.33; H, 3.52; N, 8.64. Found: C, 54.53; H, 3.55; N, 8.57; EI-MS $m / z 485.0[\mathrm{M}]^{+}$.

(Z)-4-(2-(3-chlorophenyl)-2-(2-(4-fluorophenyl)hydrazono) ethoxy)-3-(thiophen-2-yl)-1,5-dihydro-2H-pyrrol-2-one (5o) Yellow solid, m.p. $125-126{ }^{\circ} \mathrm{C}$, yield $36 \%$; IR $\left(\mathrm{KBr}, \mathrm{cm}^{-1}\right)$ : 3375, 3255, 3067, 1682; ${ }^{1} \mathrm{H}$ NMR (400 MHz, DMSO- $d_{6}$ ) $\delta 10.18(\mathrm{~s}, 1 \mathrm{H}, \mathrm{Ar}-\mathrm{NH}=\mathrm{N}), 7.97$ (s, 1H, Pyrroline-1-H), 7.89 (s, 1H, Ar(3-Cl)-2-H), 7.83 (d, J=7.7 Hz, 1H, Ar(3Cl)-6-H), $7.43(\mathrm{t}, J=6.9 \mathrm{~Hz}, 2 \mathrm{H}$, Thiophene-3,5-2H), 7.37 $(\mathrm{d}, J=7.7 \mathrm{~Hz}, 1 \mathrm{H}$, Thiophene-4-H), $7.32(\mathrm{~d}, J=5.0 \mathrm{~Hz}$, 1H, $\operatorname{Ar}(3-\mathrm{Cl})-4-\mathrm{H}), 7.29-7.22(\mathrm{~m}, 2 \mathrm{H}, \operatorname{Ar}(4-\mathrm{F})-2,6-2 \mathrm{H})$, $7.14(\mathrm{t}, J=8.6 \mathrm{~Hz}, 2 \mathrm{H}, \operatorname{Ar}(4-\mathrm{F})-3,5-2 \mathrm{H}), 6.97-6.91(\mathrm{~m}, 1 \mathrm{H}$, Ar(3-Cl)-5-H), 5.40 (s, 2H, $\mathrm{CH}_{2}$ ), 4.38 (s, 2H, Pyrroline$5-2 \mathrm{H}) ;{ }^{13} \mathrm{C}$ NMR $\left(100 \mathrm{MHz}, \mathrm{DMSO}-d_{6}\right) \delta 171.94,166.89$, $158.39,156.05,141.92,139.73,135.19,133.89,132.62$, $130.72,127.84,126.77,125.40,124.62,124.50,123.99$, 116.34, 116.11, 114.88, 114.80, 103.84, 61.62, 43.62; Anal. Calcd for $\mathrm{C}_{22} \mathrm{H}_{17} \mathrm{ClBrN}_{3} \mathrm{O}_{2} \mathrm{~S}$ (441.1): C, 59.80; H, 3.88; N, 9.51. Found: C, 59.58; H, 3.85; N, 9.57; EI-MS $m / z 441.1$ $[\mathrm{M}]^{+}$.

(Z)-4-(2-(4-fluorophenyl)-2-(2-(4-fluorophenyl)hydrazono) ethoxy)-3-(thiophen-2-yl)-1,5-dihydro-2H-pyrrol-2-one (5p) Yellow solid, m.p. $133-135{ }^{\circ} \mathrm{C}$, yield $67 \%$; IR $\left(\mathrm{KBr}, \mathrm{cm}^{-1}\right)$ : 3355, 3229, 3087, 1678; ${ }^{1} \mathrm{H}$ NMR (400 MHz, DMSO- $d_{6}$ ) $\delta 10.07(\mathrm{~s}, 1 \mathrm{H}, \mathrm{Ar}-\mathrm{NH}=\mathrm{N}), 7.98(\mathrm{~s}, 1 \mathrm{H}$, Pyrroline-1-H), 7.94-7.85 (m, 2H, Ar(4-F)-2,6-2H), 7.40 (s, 1H, Thiophene-3-H), 7.32 (d, J=4.7 Hz, 1H, Thiophene-5-H), 7.25 (d, $J=8.4 \mathrm{~Hz}, 4 \mathrm{H}, \operatorname{Ar}(4-\mathrm{F})-2,6-2 \mathrm{H}, \operatorname{Ar}(4-\mathrm{F})-3,5-2 \mathrm{H})$, $7.12(\mathrm{t}, J=8.5 \mathrm{~Hz}, 2 \mathrm{H}, \operatorname{Ar}(4-\mathrm{F})-3,5-2 \mathrm{H}), 6.94$ (s, $1 \mathrm{H}$, Thiophene-4-H), $5.40\left(\mathrm{~s}, 2 \mathrm{H}, \mathrm{CH}_{2}\right), 4.38(\mathrm{~s}, 2 \mathrm{H}$, Pyrroline$5-2 \mathrm{H}) ;{ }^{13} \mathrm{C}$ NMR $\left(100 \mathrm{MHz}, \mathrm{DMSO}-d_{6}\right) \delta 171.95,167.00$, 163.52 , 161.09, 159.09, 156.73, 142.56, 135.82, 135.76, 
$134.16,134.13,132.68,128.04,127.96,126.72,124.52$, 123.95, 117.04, 116.96, 116.91, 116.11, 115.89, 103.72, 62.11, 43.77; Anal. Calcd for $\mathrm{C}_{22} \mathrm{H}_{17} \mathrm{~F}_{2} \mathrm{~N}_{3} \mathrm{O}_{2} \mathrm{~S}$ (425.1): C, 62.11; H, 4.03; N, 9.88. Found: C, 62.49; H, 4.05; N, 9.86; EI-MS $m / z 425.1[\mathrm{M}]^{+}$.

\section{(Z)-4-(2-(4-chlorophenyl)-2-(2-(4-fluorophenyl)hydrazono)} ethoxy)-3-(thiophen-2-yl)-1,5-dihydro-2H-pyrrol-2-one (5q) Yellow solid, m.p. $131-133{ }^{\circ} \mathrm{C}$, yield $83 \%$; IR $\left(\mathrm{KBr}, \mathrm{cm}^{-1}\right)$ : 3447, 3239, 3123, 1675; ${ }^{1} \mathrm{H}$ NMR (400 MHz, DMSO$\left.d_{6}\right) \delta 10.19(\mathrm{~s}, 1 \mathrm{H}, \mathrm{Ar}-\mathrm{NH}=\mathrm{N}), 7.99(\mathrm{~s}, 1 \mathrm{H}$, Pyrroline$1-\mathrm{H}), 7.89$ (d, J=8.6 Hz, 2H, $\operatorname{Ar}(4-\mathrm{Cl})-2,6-2 \mathrm{H}), 7.46(\mathrm{~d}$, $J=8.6 \mathrm{~Hz}, 2 \mathrm{H}, \operatorname{Ar}(4-\mathrm{F})-2,6-2 \mathrm{H}), 7.41(\mathrm{~d}, J=3.2 \mathrm{~Hz}, 1 \mathrm{H}$, Thiophene-3-H), $7.32(\mathrm{~d}, J=4.7 \mathrm{~Hz}, 1 \mathrm{H}$, Thiophene-5-H), $7.27(\mathrm{dd}, J=9.0,4.8 \mathrm{~Hz}, 2 \mathrm{H}, \operatorname{Ar}(4-\mathrm{F})-3,5-2 \mathrm{H}), 7.13(\mathrm{t}$, $J=8.8 \mathrm{~Hz}, 2 \mathrm{H}, \operatorname{Ar}(4-\mathrm{Cl})-3,5-2 \mathrm{H}), 6.94(\mathrm{dd}, J=4.9,3.8 \mathrm{~Hz}$, $1 \mathrm{H}$, Thiophene-4-H), 5.41 (s, 2H, $\mathrm{CH}_{2}$ ), 4.39 (s, 2H, Pyrroline-5-2H); ${ }^{13} \mathrm{C}$ NMR (100 MHz, DMSO- $\left.d_{6}\right) \delta 171.94$, $166.89,158.30,155.96,142.05,136.47,135.44,132.69$, 132.63, 128.87, 127.56, 126.76, 124.61, 124.02, 116.27, 116.05, 114.76, 114.68, 103.83, 61.60, 43.65; Anal. Calcd for $\mathrm{C}_{22} \mathrm{H}_{17} \mathrm{FClN}_{3} \mathrm{O}_{2} \mathrm{~S}$ (441.1): C, 59.80; H, 3.88; N, 9.51. Found: C, 60.19; H, 3.90; N, 9.46; EI-MS $m / z 441.1$ [M] $^{+}$.

\section{(Z)-4-(2-(4-bromophenyl)-2-(2-(4-fluorophenyl)hydrazono)} ethoxy)-3-(thiophen-2-yl)-1,5-dihydro-2H-pyrrol-2-one (5r) White solid, m.p. $136-138{ }^{\circ} \mathrm{C}$, yield $88 \%$; IR $\left(\mathrm{KBr}, \mathrm{cm}^{-1}\right)$ : 3294, 3223, 3079, 1679; ${ }^{1} \mathrm{H}$ NMR (400 MHz, DMSO- $d_{6}$ ) $\delta 10.16(\mathrm{~s}, 1 \mathrm{H}, \mathrm{Ar}-\mathrm{NH}=\mathrm{N}), 7.98$ (s, 1H, Pyrroline-1-H), 7.91 (dd, $J=8.6,5.6 \mathrm{~Hz}, 2 \mathrm{H}, \operatorname{Ar}(4-\mathrm{Br})-2,6-2 \mathrm{H}), 7.43$ (d, $J=8.7 \mathrm{~Hz}, 2 \mathrm{H}, \operatorname{Ar}(4-\mathrm{F})-2,6-2 \mathrm{H}), 7.40(\mathrm{~d}, J=3.2 \mathrm{~Hz}, 1 \mathrm{H}$, Thiophene-3-H), 7.32 (d, J=4.9 Hz, 1H, Thiophene$5-\mathrm{H}), \quad 7.28-7.18$ (m, 4H, $\operatorname{Ar}(4-\mathrm{F})-3,5-2 \mathrm{H}, \quad \operatorname{Ar}(4-\mathrm{Br})-$ 3,5-2H), 6.96-6.90 (m, 1H, Thiophene-4-H), 5.40 (s, 2H, $\mathrm{CH}_{2}$ ), 4.37 (s, 2H, Pyrroline-5-2H); ${ }^{13} \mathrm{C}$ NMR (100 MHz, DMSO- $\left.d_{6}\right) \delta 171.95,166.88,163.58,161.14,158.19$, $155.85,142.22,142.21,135.94,134.07,132.62,128.02$, $127.94,126.76,124.61,124.03,116.25,116.03,115.85$, 115.63, 114.64, 114.56, 103.84, 61.81, 43.63; Anal. Calcd for $\mathrm{C}_{22} \mathrm{H}_{17} \mathrm{FBrN}_{3} \mathrm{O}_{2} \mathrm{~S}$ (485.0): C, 54.33; $\mathrm{H}, 3.52 ; \mathrm{N}, 8.64$. Found: C, 54.62; H, 3.54; N, 8.62; EI-MS $m / z 485.0[\mathrm{M}]^{+}$.

\section{(Z)-4-(2-(2,4-dichlorophenyl)-2-(2-(4-fluorophenyl)hydra-} zono)ethoxy)-3-(thiophen-2-yl)-1,5-dihydro-2H-pyrrol-2-one (5s)

Yellow solid, m.p. $155-157{ }^{\circ} \mathrm{C}$, yield 77\%; IR (KBr, $\left.\mathrm{cm}^{-1}\right)$ : $3431,3255,3103,1672 ;{ }^{1} \mathrm{H}$ NMR (400 MHz, DMSO- $\left.d_{6}\right) \delta$ 9.88 (s, 1H, Ar-NH=N), 7.94 (s, 1H, Pyrroline-1-H), 7.67 $(\mathrm{d}, J=2.0 \mathrm{~Hz}, 1 \mathrm{H}, \operatorname{Ar}(2,4-2 \mathrm{Cl})-3-\mathrm{H}), 7.61(\mathrm{~d}, J=8.3 \mathrm{~Hz}$, $1 \mathrm{H}$, Thiophene-3-H), 7.51 (dd, $J=8.3,2.0 \mathrm{~Hz}, 1 \mathrm{H}$, Thiophene-5-H), 7.30 (d, J=5.0 Hz, $1 \mathrm{H}, \operatorname{Ar}(2,4-2 \mathrm{Cl})-5-\mathrm{H})$, 7.18-7.06 (m, 4H, $\operatorname{Ar}(2,4-2 \mathrm{Cl})-6-\mathrm{H}, \operatorname{Ar}(4-\mathrm{F})-2,3,5-3 \mathrm{H})$, $7.04(\mathrm{~d}, J=3.0 \mathrm{~Hz}, 1 \mathrm{H}, \operatorname{Ar}(4-\mathrm{F})-6-\mathrm{H}), 6.85$ (dd, $J=5.0$,
$3.8 \mathrm{~Hz}, 1 \mathrm{H}$, Thiophene-4-H), $5.36\left(\mathrm{~s}, 2 \mathrm{H}, \mathrm{CH}_{2}\right), 4.26(\mathrm{~s}$, $2 \mathrm{H}$, Pyrroline-5-2H); ${ }^{13} \mathrm{C}$ NMR (100 MHz, DMSO- $\left.d_{6}\right)$ $\delta$ 171.81, 165.98, 158.28, 155.94, 142.19, 138.08, 135.88, $133.82,133.74,132.93,132.34,129.47,127.85,126.45$, 124.63, 124.01, 116.19, 115.97, 114.70, 114.63, 103.99, 65.77, 43.51; Anal. Calcd for $\mathrm{C}_{22} \mathrm{H}_{16} \mathrm{FCl}_{2} \mathrm{~N}_{3} \mathrm{O}_{2} \mathrm{~S}$ (475.0): C, 55.47; H, 3.39; N, 8.82. Found: C, 55.42; H, 3.36; N, 8.76; EI-MS $m / z 475.0[\mathrm{M}]^{+}$.

(Z)-4-(2-(4-methoxyphenyl)-2-(2-(4-fluorophenyl)hydrazono) ethoxy)-3-(thiophen-2-yl)-1,5-dihydro-2H-pyrrol-2-one (5t) Yellow solid, m.p. $153-155^{\circ} \mathrm{C}$, yield $58 \%$; IR $\left(\mathrm{KBr}, \mathrm{cm}^{-1}\right)$ : 3419, 3251, 3067, 1677; ${ }^{1} \mathrm{H}$ NMR (400 MHz, DMSO- $d_{6}$ ) $\delta 9.91(\mathrm{~s}, 1 \mathrm{H}, \mathrm{Ar}-\mathrm{NH}=\mathrm{N}), 7.97(\mathrm{~s}, 1 \mathrm{H}$, Pyrroline-1-H), $7.80\left(\mathrm{~d}, J=8.8 \mathrm{~Hz}, 2 \mathrm{H}, \operatorname{Ar}\left(4-\mathrm{OCH}_{3}\right)-2,6-2 \mathrm{H}\right), 7.42(\mathrm{~d}$, $J=3.6 \mathrm{~Hz}, 1 \mathrm{H}$, Thiophene-3-H), $7.32(\mathrm{~d}, J=5.0 \mathrm{~Hz}, 1 \mathrm{H}$, Thiophene-5-H), 7.22 (dd, $J=9.0,4.8 \mathrm{~Hz}, 2 \mathrm{H}, \operatorname{Ar}(4-\mathrm{F})-$ 2,6-2H), $7.11(\mathrm{t}, J=8.8 \mathrm{~Hz}, 2 \mathrm{H}, \operatorname{Ar}(4-\mathrm{F})-3,5-2 \mathrm{H}), 6.99-$ $6.92\left(\mathrm{~m}, 3 \mathrm{H}, \mathrm{Ar}\left(4-\mathrm{OCH}_{3}\right)-3,5-2 \mathrm{H}\right.$, Thiophene-4- $\left.\mathrm{H}\right), 5.38$ (s, 2H, $\mathrm{CH}_{2}$ ), 4.37 (s, 2H, Pyrroline-5-2H), $3.78(\mathrm{~s}, 3 \mathrm{H}$, $\left.\mathrm{CH}_{3}\right) ;{ }^{13} \mathrm{C}$ NMR $\left(100 \mathrm{MHz}, \mathrm{DMSO}-d_{6}\right) \delta 171.97,166.96$, $159.61,142.49,136.88,132.64,130.14,127.32,126.77$, 124.59, 124.07, 116.19, 115.97, 114.41, 114.32, 103.80, 61.89, 55.64, 43.64; Anal. Calcd for $\mathrm{C}_{23} \mathrm{H}_{20} \mathrm{FN}_{3} \mathrm{O}_{2} \mathrm{~S}$ (437.1): C, 63.15; H, 4.61; N, 9.61. Found: C, 63.42; H, 4.63; N, 9.66; EI-MS $m / z 437.1[\mathrm{M}]^{+}$.

\section{(Z)-4-(2-(4-fluorophenyl)-2-(2-(4-fluorophenyl)hydrazono) ethoxy)-1-methyl-3-(thiophen-2-yl)-1,5-dihydro-2H-pyr- rol-2-one (5u)}

White solid, m.p. $147-149{ }^{\circ} \mathrm{C}$, yield $80 \%$; IR $\left(\mathrm{KBr}, \mathrm{cm}^{-1}\right)$ : 3263, 2987, 1667; ${ }^{1} \mathrm{H}$ NMR (400 MHz, DMSO- $d_{6}$ ) $\delta 10.12$ $(\mathrm{s}, 1 \mathrm{H}, \mathrm{Ar}-\mathrm{NH}=\mathrm{N}), 7.89(\mathrm{dd}, J=8.3,5.7 \mathrm{~Hz}, 2 \mathrm{H}, \operatorname{Ar}(4-\mathrm{F})-$ 2,6-2H), 7.41 (d, $J=2.8 \mathrm{~Hz}, 1 \mathrm{H}$, Thiophene-5-H), 7.32 (d, $J=4.9 \mathrm{~Hz}, 1 \mathrm{H}$, Thiophene-3-H), $7.25(\mathrm{dt}, J=13.7,6.8 \mathrm{~Hz}$, 4H, $\operatorname{Ar}(4-\mathrm{F})-3,5-2 \mathrm{H}, \operatorname{Ar}(4-\mathrm{F})-2,6-2 \mathrm{H}), 7.12(\mathrm{t}, J=8.8 \mathrm{~Hz}$, 2H, $\operatorname{Ar}(4-\mathrm{F})-3,5-2 \mathrm{H}), 6.96-6.91$ (m, 1H, Thiophene-4-H), 5.41 (s, 2H, $\mathrm{CH}_{2}$ ), 4.47 (s, 2H, Pyrroline-5-2H), 2.99 (s, $\left.3 \mathrm{H}, \mathrm{CH}_{3}\right) ;{ }^{13} \mathrm{C}$ NMR (100 MHz, DMSO-d $) \delta 169.51$, $164.59,163.57,161.13,158.18,155.84,142.29,135.78$, $134.15,132.64,128.01,127.93,126.83,124.67,124.06$, $116.22,116.00,115.83,115.62,114.65,114.58,103.65$, 62.08, 49.70, 29.06; Anal. Calcd for $\mathrm{C}_{23} \mathrm{H}_{19} \mathrm{~F}_{2} \mathrm{~N}_{3} \mathrm{O}_{2} \mathrm{~S}$ (439.1): C, 62.86; H, 4.36; N, 9.56. Found: C, 62.51; H, 4.39 ; N, 9.52; EI-MS $m / z 439.1[\mathrm{M}]^{+}$.

(Z)-4-(2-(4-fluorophenyl)-2-(2-(4-methylphenyl)hydrazono) ethoxy)-1-methyl-3-(thiophen-2-yl)-1,5-dihydro-2H-pyrrol-2-one (5v)

White solid, m.p. $157-159{ }^{\circ} \mathrm{C}$, yield $53 \%$; IR $\left(\mathrm{KBr}, \mathrm{cm}^{-1}\right)$ : 3257, 2922, 1670; ${ }^{1} \mathrm{H}$ NMR (400 MHz, DMSO- $d_{6}$ ) $\delta 10.08$ $(\mathrm{s}, 1 \mathrm{H}, \mathrm{Ar}-\mathrm{NH}=\mathrm{N}), 7.76\left(\mathrm{~d}, J=7.7 \mathrm{~Hz}, 2 \mathrm{H}, \mathrm{Ar}\left(4-\mathrm{CH}_{3}\right)-\right.$ 2,6-2H), 7.41 (s, $1 \mathrm{H}$, Thiophene-5-H), $7.31(\mathrm{~d}, J=8.5 \mathrm{~Hz}$, 
$3 \mathrm{H}$, Thiophene-3-H, $\left.\operatorname{Ar}\left(4-\mathrm{CH}_{3}\right)-3,5-2 \mathrm{H}\right), 7.28-7.17(\mathrm{~m}$, 4H, $\operatorname{Ar}(4-\mathrm{F})-2,3,5,6-4 \mathrm{H}), 6.93$ (s, 1H, Thiophene-4-H), $5.37\left(\mathrm{~s}, 2 \mathrm{H}, \mathrm{CH}_{2}\right), 4.45$ (s, 2H, Pyrroline-5-2H), 2.99 $\left(\mathrm{s}, 3 \mathrm{H}, \mathrm{CH}_{3}\right), 2.32\left(\mathrm{~s}, 3 \mathrm{H}, \mathrm{CH}_{3}\right) ;{ }^{13} \mathrm{C}$ NMR $(100 \mathrm{MHz}$, DMSO- $\left.d_{6}\right) \delta 169.53,164.71,158.08,155.74,142.40$, $137.58,136.59,134.88,132.67,129.49,126.82,125.80$, $124.63,124.07,116.96,116.88,116.19,115.97,114.55$, 114.48, 103.59, 62.06, 49.71, 29.05, 21.26; Anal. Calcd for $\mathrm{C}_{24} \mathrm{H}_{22} \mathrm{FN}_{3} \mathrm{O}_{2} \mathrm{~S}$ (435.1): C, 66.19; H, 5.09; N, 9.65. Found: C, 66.44; H, 5.12; N, 9.71; EI-MS m/z $435.1[\mathrm{M}]^{+}$.

\section{(Z)-4-(2-(4-methylphenyl)-2-(2-(4-methylphenyl)hydrazono) ethoxy)-1-methyl-3-(thiophen-2-yl)-1,5-dihydro-2H-pyr- rol-2-one (5w)}

White solid, m.p. $175-177^{\circ} \mathrm{C}$, yield $41 \%$; IR $\left(\mathrm{KBr}, \mathrm{cm}^{-1}\right)$ : 3230, 2988, 1668; ${ }^{1} \mathrm{H}$ NMR (400 MHz, DMSO- $\left.d_{6}\right) \delta 9.99$ $(\mathrm{s}, 1 \mathrm{H}, \operatorname{Ar}-\mathrm{NH}=\mathrm{N}), 7.74\left(\mathrm{~d}, J=8.2 \mathrm{~Hz}, 2 \mathrm{H}, \operatorname{Ar}\left(4-\mathrm{CH}_{3}\right)-\right.$ 3,5-2H), 7.43-7.39 (m, 1H, Thiophene-5-H), 7.31 (dd, $J=5.1,0.9 \mathrm{~Hz}, 1 \mathrm{H}$, Thiophene-3-H), $7.21(\mathrm{~d}, J=8.1 \mathrm{~Hz}$, $\left.2 \mathrm{H}, \operatorname{Ar}\left(4-\mathrm{CH}_{3}\right)-2,6-2 \mathrm{H}\right), 7.16(\mathrm{~d}, J=8.4 \mathrm{~Hz}, 2 \mathrm{H}, \operatorname{Ar}(4-$ $\left.\left.\mathrm{CH}_{3}\right)-2,6-2 \mathrm{H}\right), 7.07$ (d, J=8.4 Hz, $\left.2 \mathrm{H}, \mathrm{Ar}\left(4-\mathrm{CH}_{3}\right)-3,5-2 \mathrm{H}\right)$, $6.93(\mathrm{dd}, J=5.1,3.7 \mathrm{~Hz}, 1 \mathrm{H}$, Thiophene-4-H), $5.40(\mathrm{~s}, 2 \mathrm{H}$, $\mathrm{CH}_{2}$ ), 4.49 (s, 2H, Pyrroline-5-2H), 2.99 (s, 3H, $\mathrm{CH}_{3}$ ), $2.32\left(\mathrm{~s}, 3 \mathrm{H}, \mathrm{CH}_{3}\right), 2.23\left(\mathrm{~s}, 3 \mathrm{H}, \mathrm{CH}_{3}\right) ;{ }^{13} \mathrm{C}$ NMR $(100 \mathrm{MHz}$, DMSO- $\left.d_{6}\right) \delta 169.56,164.84,143.52,137.32,135.62$, $135.08,132.70,129.96,129.46,128.77,126.82,125.66$, 124.57, 124.02, 113.48, 103.49, 62.06, 49.75, 29.06, 21.26, 20.75; Anal. Calcd for $\mathrm{C}_{25} \mathrm{H}_{25} \mathrm{~N}_{3} \mathrm{O}_{2} \mathrm{~S}$ (431.1): C, 69.58; $\mathrm{H}$, 5.84; N, 9.74. Found: C, 69.36; H, 5.87; N, 9.77; EI-MS $m / z 431.1[\mathrm{M}]^{+}$.

\section{Conclusions}

A series of 3-(thiophen-2-yl)-1,5-dihydro- $2 \mathrm{H}$-pyrrol2-one derivatives bearing a hydrazone group were designed, synthesized and confirmed by FT-IR, ${ }^{1} \mathrm{H}$ NMR, ${ }^{13} \mathrm{C}$ NMR, EI-MS, NOESY and elemental analysis. The antifungal assays indicated that some the title compounds exhibited obvious antifungal activity against $F g$, $R s, B c$ and $C c$. Strikingly, the $\mathrm{EC}_{50}$ value of $5 \mathbf{e}$ against $R s$ was $1.26 \mu \mathrm{g} / \mathrm{mL}$, which is better than that of drazoxolon $(1.77 \mu \mathrm{g} / \mathrm{mL})$. Meanwhile, title compounds $\mathbf{5 b}, \mathbf{5 d}, \mathbf{5 e}-$ $\mathbf{5 g}, \mathbf{5 n}-\mathbf{5 q}$ and $5 \mathbf{t}$ exhibited remarkable anti- $C c$ activity, with corresponding $\mathrm{EC}_{50}$ values reached 5.52-9.97 $\mu \mathrm{g} /$ $\mathrm{mL}$, which are better than that of drazoxolon $(19.46 \mu \mathrm{g} /$ $\mathrm{mL}$ ). These results indicated that 3-(thiophen-2-yl)1,5-dihydro-2 $H$-pyrrol-2-one derivatives containing a hydrazone group can serve as potential structural templates in the search for novel and highly efficient fungicides. Further studies on the antifungal mechanism and structural modification of 3-(thiophen-2-yl)-1,5-dihydro- $2 \mathrm{H}$-pyrrol-2-one derivatives containing a hydrazone group are currently underway.

\section{Additional file}

Additional file 1. All the copies of FT-IR, ${ }^{1} \mathrm{H}$ NMR, ${ }^{13} \mathrm{C}$ NMR and El-MS for title compounds $\mathbf{5 a}-\mathbf{5 w}$

\section{Authors' contributions}

The current study is an outcome of constructive discussion with CY. ZR, XW, $M C$ and $M W$ carried out the synthesis and characterization experiments of title compounds; ZR, MC, MW and XW tested the antifungal activity of target compounds; XW, ZR, MC and AL performed the FT-IR, ${ }^{1} \mathrm{H} \mathrm{NMR},{ }^{13} \mathrm{C} N M R, E I-M S$, NOESY and elemental analyses; $X W$ and $C Y$ were involved in the drafting of the manuscript and revising the manuscript. All authors read and approved the final manuscript.

\section{Author details}

1 Jiangsu Key Laboratory of Pesticide Science, College of Sciences, Nanjing Agricultural University, Nanjing 210095, China. ${ }^{2}$ Key Laboratory of Monitoring and Management of Crop Diseases and Pest Insects, Ministry of Agriculture, Nanjing Agricultural University, Nanjing 210095, China.

\section{Acknowledgements}

The authors gratefully acknowledge the grants from the National Nature Science Foundation of China (No. 31772209) and the Fundamental Research Funds for the Central Universities of China (No. KYTZ201604).

\section{Competing interests}

The authors declare that they have no competing interests.

\section{Availability of data and materials}

We have presented all our main data in the form of tables and figures. All the copies of IR, ${ }^{1} \mathrm{H}$ NMR, ${ }^{13} \mathrm{C}$ NMR and El-MS spectrogram for title compounds $\mathbf{5 a}-\mathbf{5} \mathbf{w}$ were presented in the Additional file 1. The datasets supporting the conclusions of the article are included within the article and the Additional file 1.

\section{Consent for publication}

This section are not applicable for this manuscript.

\section{Ethics approval and consent to participate}

This section are not applicable for this manuscript.

\section{Publisher's Note}

Springer Nature remains neutral with regard to jurisdictional claims in published maps and institutional affiliations.

Received: 12 June 2018 Accepted: 10 July 2018

Published online: 17 July 2018

\section{References}

1. Sparks TC, Lorsbach BA (2017) Perspectives on the agrochemical industry and agrochemical discovery. Pest Manag Sci 73:672-677

2. Swain T (1977) Secondary compounds as protective agents. Ann Rev Plant Physiol 28:479-501

3. Qian X, Lee PW, Cao S (2010) China: forward to the green pesticides via basic research program. J Agric Food Chem 58:2613-2623

4. Schobert R, Schlenk A (2008) Tetramic and tetronic acids: an update on new derivatives and biological aspects. Bioorg Med Chem 16:4203-4221

5. Jeong YC, Moloney MG (2009) Tetramic acids as scaffolds: synthesis, tautomeric and antibacterial behaviour. Synlett 15:2487-2491

6. Lu GH, Chu HB, Chen M, Yang CL (2014) Synthesis and bioactivity of novel strobilurin derivatives containing pyrrolidine-2,4-dione moiety. Chin Chem Lett 25:61-64

7. Han BF, Shi QM, Wang XF, Liu JB, Qiang S, Yang CL (2012) Synthesis and bioactivity of novel 3-(1-hydroxyethylidene)-5-substituted-pyrrolidine2,4-dione derivatives. Chin Chem Lett 23:1023-1026 
8. Bruck E, Elbert A, Fischer R, Krueger S, Kuhnhold J, Klueken AM, Nauen R, Niebes JF, Reckmann U, Schnorbach HJ, Steffens R, Waetermeulen XV (2009) Movento, an innovative ambimobile insecticide for sucking insect pest control in agriculture: biological profile and filed performance. Crop Prot 28:838-844

9. Wang PY, Chen L, Zhou J, Fang HS, Wu ZB, Song BA, Yang S (2017) Synthesis and bioactivities of 1-ary-4-hydroxy-1H-pyrrol-2(5H)-one derivatives bearing 1,3,4-oxadiazole moiety. J Saudi Chem Soc 21:315-323

10. Wang XF, Si TF, Li QB, Zhu ZY, Zhu XJ, Qiang S, Yang CL (2010) Synthesis, characterization and biological activity of novel $(5-R S, 6 S)-5$-sec-butyl3-(1-substitutes amino)ethylidene-1H-pyrrilidine-2,4-diones. ARKIVOC 2:31-48

11. Zhang L, Ren Z, Lu A, Zhao Z, Xu W, Bao Q, Ding W, Yang C (2015) Synthesis, biological activity and 3D-QSAR study of novel pyrrolidine-2,4-dione derivatives containing $N$-substituted phenylhydrazine moiety. Chem Res Chin Univ 31:228-234

12. Zhu X, Huang L, Wang X, Zhu Z, Zheng X, Qiang S, Yang C (2009) Synthesis and biological activities of 3-(1'-alkyloxyiminoethyl)-4-hydroxypyrroline-2-one derivatives. Chin J Org Chem 29:1784-1789

13. Xu WQ, Chen M, Wang KY, Ren ZJ, Lu AM, Yang CL (2016) Synthesis, characterization, and antifungal activity of phenylpyrrole-substituted tetramic acids bearing carbonates. Molecules 21:355

14. Zhong B, Li ZM, Liu CL, Zhao WG (2004) Synthesis and biological activity of polysubstituted pyridines. Chin J Org Chem 24:1304-1306

15. Harit T, Bellaouchi R, Asehraou A, Rahal M, Bouabdallah I, Malek F (2017) Synthesis, characterization, antimicrobial activity and theoretical studies of new thiophene-based tripodal ligands. J Mol Struct 1133:74-79

16. Mabkhot YN, Alatibi F, El-Sayed NNE, Kheder NA, Al-Showiman SS (2016) Synthesis and structure-activity relationship of some new thiophenebased heterocycles as potential antimicrobial agents. Molecules 21:1036

17. Mabkhot YN, Kaal NA, Alterary S, Al-Showiman SS, Farghaly TA, Mubarak MS (2017) Antimicrobial activity of thiophene derivatives derived from ethyl (E)-5-(3-(dimethylamino)acryloyl)-4-methyl-2-(phenylamino) thiophene-3-carboxylate. Chem Cent J 11:75

18. Yang ZB, Hu DY, Zeng S, Song BA (2016) Novel hydrazone derivatives containing pyridine amide moiety: design, synthesis, and insecticidal activity. Bioorg Med Chem Lett 26:1161-1164

19. Wang Z, Xie D, Gan X, Zeng S, Zhang A, Yin L, Song B, Jin L, Hu D (2017) Synthesis, antiviral activity, and molecular docking study of trans-ferulic acid derivatives containing acylhydrazone moiety. Bioorg Med Chem Lett 27:4096-4100

20. Backes GL, Neumann DM, Jursic BS (2014) Synthesis and antifungal activity of substituted salicylaldehyde hydrazones, hydrazides and sulfohydrazides. Bioorg Med Chem 22:4629-4636
21. Aggarwal N, Kumar R, Srivastva C, Dureja P, Khurana JM (2010) Synthesis of nalidixic acid based hydrazones as novel pesticides. J Agric Food Chem 58:3056-3061

22. Liu Y, Song H, Huang Y, Li J, Zhao S, Song Y, Yang P, Xiao Z, Liu Y, Li Y, Shang $H$, Wang Q (2014) Design, synthesis, and antiviral, fungicidal, and insecticidal activities of tetrahydro- $\beta$-carboline-3-carbohydrazide derivatives. J Agric Food Chem 62:9987-9999

23. Dai ZC, Chen YF, Zhang M, Li SK, Yang TT, Shen L, Wang JX, Qian SS, Zhu $\mathrm{HL}$, Ye YH (2015) Synthesis and antifungal activity of 1,2,3-triazole phenylhydrazone derivatives. Org Biomol Chem 13:477-486

24. Wang X, Chen YF, Yan W, Cao LL, Ye YH (2016) Synthesis and biological evaluation of benzimidazole phenylhydrazone derivatives as antifungal agents against phytopathogenic fungi. Molecules 21:1574

25. Liu J, Cui Z, He H (2012) Synthesis and pesticidal activity of 3-(2-chloro4-trifluoromethyl)phenoxy benzoylhydrazones. Chin J Org Chem 32:1925-1929

26. Wu J, Wang J, Hu D, He M, Jin L, Song B (2012) Synthesis and antifungal activity of novel pyrazolecarboxamide derivatives containing a hydrazone moiety. Chem Cent J 6:51

27. Zhang M, Dai ZC, Qian SS, Liu JY, Xiao Y, Lu AM, Zhu HL, Wang JX, Ye YH (2014) Design, synthesis, antifungal, and antioxidant activities of (E)-6-((2phenylhydrazono)methyl)quinoxaline derivatives. J Agric Food Chem 62:9637-9643

28. Wang JJ, Si WJ, Chen M, Lu AM, Zhang WH, Yang CL (2017) Synthesis and fungicidal activity of phenylhydrazone derivatives containing two carbonic acid ester groups. J Pestic Sci 42:84-92

29. Yang C, Ren Z, Shi S, Xu W, Li L, Wang K (2016) Preparation of thiophenepyrrolidone compounds for preventing and controlling plant pathogenic fungi. C.N. patent, 105218534

30. Hamdi N, Passarelli V, Romerosa A (2011) Synthesis, spectroscopy and electrochemistry of new 4-(4-acetyl-5-substituted-4,5-dihydro-1,3,4oxodiazol-2-yl)methoxy)-2H-chromen-2-ones as a novel class of potential antibacterial and antioxidant derivatives. C R Chimie 14:548-555

31. Lu S, Drees M, Yao Y, Boudinet D, Yan H, Pan H, Wang J, Li Y, Usta H, Facchetti A (2013) 3,6-Dithiophen-2-yl-diketopyrrolo[3,2-b]pyrrole (isoDPPT) as an acceptor building block for organic opto-electronics. Macromolecules 46:3895-3906

32. Yurttas L, Duran M, Demirayak S, Gencer HK, Tunali Y (2013) Synthesis and initial biological evaluation of substituted 1-phenylamino-2-thio-4,5-dimethyl-1H-imidazole derivatives. Bioorg Med Chem Lett 23:6764-6768

\section{Submit your manuscript to a SpringerOpen ${ }^{\odot}$ journal and benefit from:}

- Convenient online submission

- Rigorous peer review

- Open access: articles freely available online

- High visibility within the field

- Retaining the copyright to your article

Submit your next manuscript at $\boldsymbol{\nabla}$ springeropen.com 